ACCepted for publication in the Astrophysical Journal

Preprint typeset using LATEX style emulateapj v. 08/13/06

\title{
TYPE Ib SUPERNOVA 2008D ASSOCIATED WITH THE LUMINOUS X-RAY TRANSIENT 080109: AN ENERGETIC EXPLOSION OF A MASSIVE HELIUM STAR
}

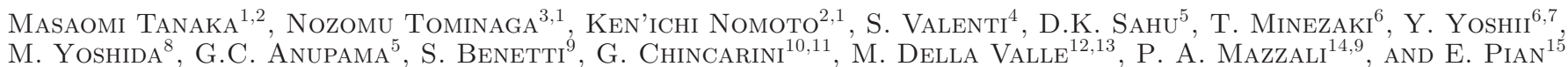

\section{ABSTRACT}

We present a theoretical model for supernova (SN) 2008D associated with the luminous X-ray transient 080109. The bolometric light curve and optical spectra of the SN are modelled based on the progenitor models and the explosion models obtained from hydrodynamic/nucleosynthetic calculations. We find that SN 2008D is a more energetic explosion than normal core-collapse supernovae, with an ejecta mass of $M_{\mathrm{ej}}=5.3 \pm 1.0 M_{\odot}$ and a kinetic energy of $E_{\mathrm{K}}=6.0 \pm 2.5 \times 10^{51} \mathrm{erg}$. The progenitor star of the $\mathrm{SN}$ has a $6-8 M_{\odot}$ He core with essentially no $\mathrm{H}$ envelope $\left(<5 \times 10^{-4} M_{\odot}\right)$ prior to the explosion. The main-sequence mass of the progenitor is estimated to be $M_{\mathrm{MS}}=20-25 M_{\odot}$, with additional systematic uncertainties due to convection, mass loss, rotation, and binary effects. These properties are intermediate between those of normal SNe and hypernovae associated with gamma-ray bursts. The mass of the central remnant is estimated as $1.6-1.8 M_{\odot}$, which is near the boundary between neutron star and black hole formation.

Subject headings: supernovae: general — supernovae: individual (SN 2008D) — nuclear reactions, nucleosynthesis, abundances — radiative transfer

\section{INTRODUCTION}

A luminous X-ray transient was discovered in NGC 2770 in the Swift XRT data taken on 2008 January 9 for the observation of SN 2007uy in the same galaxy (Berger \& Soderberg 2008; Kong \& Maccarone 2008). The Xray emission of the transient reached a peak $\sim 65 \mathrm{sec}-$ onds, lasting $\sim 600$ seconds, after the observation started (Page et al. 2008). The X-ray spectrum is soft, and no $\gamma$-ray counterpart was detected by the Swift BAT (Page et al. 2008).

${ }^{1}$ Department of Astronomy, School of Science, University of Tokyo, 7-3-1 Hongo, Bunkyo-ku, Tokyo 113-0033, Japan; mtanaka@astron.s.u-tokyo.ac.jp

${ }^{2}$ Institute for the Physics and Mathematics of the Universe, University of Tokyo, Kashiwanoha 5-1-5, Kashiwa, Chiba 277-8568, Japan

${ }^{3}$ Division of Optical and Infrared Astronomy, National Astronomical Observatory of Japan, 2-21-1 Osawa, Mitaka, Tokyo 1818588, Japan; nozomu.tominaga@nao.ac.jp

4 Astrophysics Research Centre, School of Maths and Physics, Queen's University, Belfast, BT7 1NN, Northern Ireland, UK

${ }^{5}$ Indian Institute of Astrophysics, II Block Koramangala, Bangalore 560034, India

${ }^{6}$ Institute of Astronomy, School of Science, University of Tokyo, 2-21-1 Osawa, Mitaka, Tokyo 181-0015, Japan

${ }^{7}$ Research Center for the Early Universe, School of Science, University of Tokyo, 7-3-1 Hongo, Bunkyo-ku, Tokyo 113-003, Japan

${ }^{8}$ Okayama Astrophysical Observatory, National Astronomical Observatory of Japan

9 Istituto Naz. di Astrofisica-Oss. Astron., vicolo dell'Osservatorio, 5, 35122 Padova, Italy

${ }^{10}$ Universit degli Studi di Milano Bicocca, Dipartimento di Fisica, Piazza della Scienze 3, 20126 Milano, Italy

${ }^{11}$ INAF, Osservatorio Astronomico di Brera, via E. Bianchi 46, 23807 Merate (LC), Italy

12 Capodimonte Astronomical Observatory, Salita Moiariello 16, I-80131, INAF- Napoli, Italy

13 European Southern Observatory, Karl-Schwarzschild-Strasse 2, D-85748, Garching, Germany

${ }_{14}$ Max-Planck Institut für Astrophysik, Karl-SchwarzschildStrasse 2 D-85748 Garching bei München, Germany

${ }^{15}$ Istituto Naz. di Astrofisica-Oss. Astron., Via Tiepolo, 11, 34131 Triste, Italy
Given the small total X-ray energy and the soft Xray emission, Soderberg et al. (2008) and Chevalier \& Fransson (2008) interpreted the X-ray transient as a supernova (SN) shock breakout. On the other hand, Xu et al. (2008), Li (2008) and Mazzali et al. (2008) considered this transient as the least energetic end of gamma-ray bursts (GRBs) and X-ray flashes (XRFs).

The optical counterpart was discovered at the position of the X-ray transient (Deng \& Zhu 2008; Valenti et al. 2008a), confirming the presence of a SN, named SN 2008D (Li \& Filippenko 2008). To study this event in detail, intensive follow-up observations were carried out over a wide wavelength range including $\mathrm{X}$-rays (Soderberg et al. 2008; Modjaz et al. 2008b), optical/NIR (Soderberg et al. 2008; Malesani et al. 2009; Modjaz et al. 2008b; Mazzali et al. 2008), and radio (Soderberg et al. 2008).

SN 2008D showed a broad-line optical spectrum at early epochs $(t \lesssim 10$ days, hereafter $t$ denotes time after the transient, 2008 Jan 9.56 UT; Soderberg et al. 2008). However, the spectrum changed to that of normal Type Ib SN, i.e., SN with $\mathrm{He}$ absorption lines and without $\mathrm{H}$ lines (Modjaz et al. 2008c). To date, the SN associated with GRBs or XRFs are all Type Ic, i.e., SNe without $\mathrm{H}$ and He absorption.

Soderberg et al. (2008) and Mazzali et al. (2008) estimated the ejected mass and the kinetic energy of SN 2008D. Using analytic formulae, Soderberg et al. (2008) suggested that this SN has the ejecta mass $M_{\mathrm{ej}}=$ $3-5 M_{\odot}$ and the kinetic energy of the ejecta $E_{\mathrm{K}} \sim$ $2-4 \times 10^{51} \mathrm{erg}$. Mazzali et al. (2008) did model calculations and suggested that this event is intermediate between normal SNe and GRB-associated SNe (or hypernovae), with $M_{\mathrm{ej}} \sim 7 M_{\odot}$ and $E_{\mathrm{K}} \sim 6 \times 10^{51} \mathrm{erg}$. In their model calculations, hydrodynamic/nucleosynthetic models are not used, and thus, their estimate of the core mass prior to the explosion and the progenitor main-sequence 
TABLE 1

EXPLOSION MODELS

\begin{tabular}{|c|c|c|c|c|c|c|c|c|c|c|}
\hline Model & $M_{\mathrm{MS}}{ }^{\mathrm{a}}$ & $M_{\alpha}^{\mathrm{b}}$ & $R_{*}{ }^{\mathrm{c}}$ & $M_{\text {cut }}{ }^{\mathrm{d}}$ & $M_{\mathrm{ej}}{ }^{\mathrm{e}}$ & $E_{\mathrm{K}}{ }^{\mathrm{f}}$ & $M\left({ }^{56} \mathrm{Ni}\right)^{\mathrm{g}}$ & $v_{\mathrm{He}}{ }^{\mathrm{h}}$ & $v_{\mathrm{Ni}}{ }^{\mathrm{i}}$ & $M_{0.1 c}{ }^{\mathrm{j}}$ \\
\hline $\mathrm{HE} 4$ & $\approx 15$ & 4 & 3.5 & 1.3 & 2.7 & 1.1 & 0.07 & $<3500$ & 7900 & $<3.0 \times 10^{-5}$ \\
\hline HE6 & $\approx 20$ & 6 & 2.2 & 1.6 & 4.4 & 3.7 & 0.065 & 6700 & 7000 & 0.007 \\
\hline $\mathrm{HE} 8$ & $\approx 25$ & 8 & 1.3 & 1.8 & 6.2 & 8.4 & 0.07 & 10500 & 9000 & 0.04 \\
\hline HE10 & $\approx 30$ & 10 & 1.2 & 2.3 & 7.7 & 13.0 & 0.07 & 12500 & 10600 & 0.09 \\
\hline HE16 & $\approx 40$ & 16 & 0.74 & 3.6 & 12.4 & 26.5 & 0.07 & 17500 & 14000 & 0.24 \\
\hline Soderberg et al. (2008) & & & $\sim 1^{\mathrm{k}}$ & & $3-5$ & $2-4$ & 0.05 & & & \\
\hline Mazzali et al. (2008) & $\sim 30$ & & & & $\sim 7$ & $\sim 6$ & 0.09 & & & 0.03 \\
\hline
\end{tabular}

${ }^{a}$ Main-sequence mass $\left(M_{\odot}\right)$ estimated from the approximate formula obtained by Sugimoto \& Nomoto $(1980)$

b The mass of the He star $\left(M_{\odot}\right)$

${ }^{\mathrm{c}}$ Progenitor radius prior to the explosion $\left(R_{\odot}\right)$

d Mass cut $\left(M_{\odot}\right)$

e The mass of the $\mathrm{SN}$ ejecta $\left(M_{\odot}\right)$

${ }^{\mathrm{f}} \mathrm{The}$ kinetic energy of the SN ejecta $\left(10^{51} \mathrm{erg}\right)$

g The mass of ejected ${ }^{56} \mathrm{Ni}\left(M_{\odot}\right)$

${ }^{\mathrm{h}}$ Velocity at the bottom of the He layer $\left(\mathrm{km} \mathrm{s}^{-1}\right)$

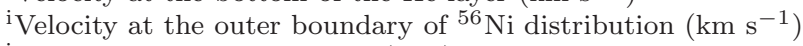

${ }^{\mathrm{j}}$ The ejecta mass at $v>0.1 c\left(M_{\odot}\right)$

${ }^{\mathrm{k}}$ Estimated from the photospheric radius and temperature of the early part of the LC ( $t \lesssim 4$ days) using the formulae by Waxman et al. (2007). This is consistent with the estimate by Modjaz et al. (2008b) while Chevalier \& Fransson $(2008)$ derived $\sim 9 R_{\odot}$ using $M_{\text {ej }}$ and $E_{\mathrm{K}}$ estimated by Soderberg et al. (2008) and the formulae by Chevalier (1992).

mass is less direct.

In this paper, we present detailed theoretical study of emission from SN 2008D. The bolometric light curve (LC) and optical spectra are modelled based on the progenitor models and the explosion models obtained from hydrodynamic/nucleosynthetic calculations. In $\S 2$, we show the progenitor and explosion models. The optical LC and spectra are modelled in $\S 3$ and $\S 4$, respectively. We discuss the nature of SN 2008D in $\S 5$ and finally give conclusions in $\S 6$. Throughout this paper, we adopt $31 \mathrm{Mpc}(\mu=32.46 \mathrm{mag})$ for the distance to $\mathrm{SN}$ 2008D (Modjaz et al. 2008b; Mazzali et al. 2008) and $E(B-V)=0.65 \mathrm{mag}$ for the total reddening (Mazzali et al. 2008).

\section{MODELS}

To understand the nature of SN 2008D and its progenitor star, (1) we first construct the exploding $\mathrm{He}$ star models with various masses by performing hydrodynamic/nucleosynthetic calculations for the presupernova He star models. (2) The important parameters of the SN, such as the mass $\left(M_{\mathrm{ej}}\right)$ and kinetic energy of the ejecta $\left(E_{\mathrm{K}}\right)$, are estimated by modelling the bolometric LC (§3) and the optical spectra (§4). Then, (3) the He star mass $\left(M_{\alpha}\right)$ can be estimated from the best set of $M_{\mathrm{ej}}$ and $E_{\mathrm{K}}$. Finally, (4) the main-sequence mass $\left(M_{\mathrm{MS}}\right)$ of the progenitor star can be estimated by the evolution models, which predict the $M_{\mathrm{MS}}-M_{\alpha}$ relation.

In this section, we construct hydrodynamic models using an evolutionary model. The progenitor model and hydrodynamic/nucleosynthetic calculations are described in $\S 2.1$ and $\S 2.2$, respectively. The hydrodynamic models are tested in $\S 3$ and $\S 4$.

\subsection{Progenitor Models}

In the strategy described above, presupernova He star models are required as input for the hydrodynamic calculations. The Wolf-Rayet star models with stellar winds tend to form the He stars whose masses are larger than that inferred for SN 2008D (e.g., Soderberg et al. 2008).
To study the properties of the ejecta and the progenitor star without specifying the mass loss mechanism (stellar winds in Wolf-Rayet star or Roche lobe overflow in close binary, i.e., possible binary progenitor scenario, e.g., Wellstein \& Langer 1999), we adopt the He star evolution models with various masses. We use five $\mathrm{He}$ star models with the masses of $4,6,8,10$ and $16 M_{\odot}$ (Nomoto \& Hashimoto 1988; Nomoto et al. 1997; Nakamura et al. 2001b), where the mass loss is not taken into account. These models are called HE4, HE6, HE8, HE10 and HE16, respectively. The corresponding mainsequence masses of these models are $\approx 15,20,25,30$ and $40 M_{\odot}$, respectively (Table 1 ), which is estimated from the approximate formula of the $M_{\mathrm{MS}}-M_{\alpha}$ relation obtained by Sugimoto \& Nomoto (1980; Eq. 4.1).

The difference in the density structure of the He core is negligible among different stellar evolutionary calculations if the $\mathrm{He}$ core mass is the same. As a result, the observable quantities (i.e., LC and spectra) after the hydrodynamic and radiative transfer calculations are not affected by the variety of the evolutionary models. Thus, the estimate of $M_{\mathrm{ej}}$ and $E_{\mathrm{K}}$ does not depend on the evolutionary models.

However, the $M_{\mathrm{MS}}-M_{\alpha}$ relation depends on the several evolutionary processes that are subject to uncertainties, e.g., the convective overshooting, wind mass loss, shear mixing and meridional circulation in rotating stars. The different assumptions adopted in different stellar calculation codes may affect the $M_{\mathrm{MS}}-M_{\alpha}$ relation as summarized in Figure 1. The red line shows the formula derived by Sugimoto \& Nomoto (1980). The other lines show the relations obtained by the models including mass loss (Limongi \& Chieffi 2006, blue; Hirschi et al. 2004, green; Rauscher et al. 2002, black), and rotation $\left(v_{Z A M S}=300\right.$ $\mathrm{km} \mathrm{s}^{-1}$; Hirschi et al. 2004, cyan). The models shown in open squares have a $\mathrm{H}$ envelope prior to the explosion while the models shown in the filled squares have a bare He core.

These models assume the solar abundance for the initial abundance. The mass loss causes the smaller $M_{\alpha}$ 


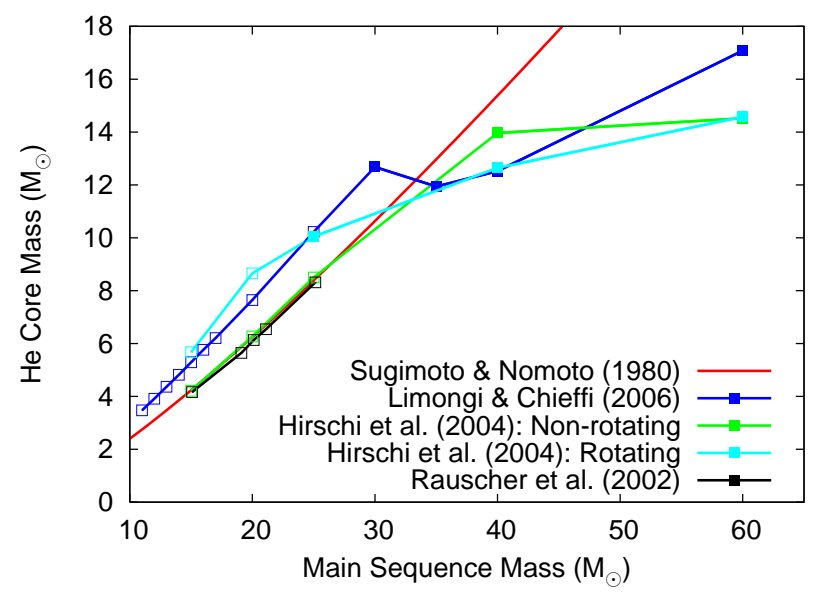

FIG. 1.- Relation between the main-sequence mass and the $\mathrm{He}$ core mass in the formula derived by Sugimoto \& Nomoto (1980; red, used in Nomoto \& Hashimoto 1988). It is compared with the relation between the main-sequence mass and the He core mass at the presupernova stage in the models by Limongi \& Chieffi (2006, blue), Hirschi et al. (2004, green and cyan for non-rotating and rotating models, respectively), and Rauscher et al. (2002, black) The models shown in open squares have $\mathrm{H}$ envelope while the models shown in filled squares have a bare He core.

for $M_{\mathrm{MS}}>30 M_{\odot}$ in the models by Limongi \& Chieffi (2006) and Hirschi et al. (2004). For the lower metallicity models, the mass loss rate is lower, so that the $M_{\mathrm{MS}}-M_{\alpha}$ relation would be closer to that of Sugimoto \& Nomoto (1980). For the stars with $M_{\mathrm{MS}} \lesssim 30 M_{\odot}$ or $M_{\alpha} \lesssim 10 M_{\odot}$, the gradient in the plot is similar among the models.

At the presupernova stage, the He stars consist of the Fe core, Si-rich layer, O-rich layer, and He-rich layer. The mass of the Fe-core is $\sim 1.4-1.6 M_{\odot}$, depending on the model. The mass of the O-rich layer is sensitive to the progenitor mass, while the mass of the He-rich layer is $\sim 2 M_{\odot}$ irrespectively of the He star mass. Note that the mass of He-rich layer can be as large as $\sim 3 M_{\odot}$ depending on the evolutionary models (e.g., Limongi \& Chieffi 2006) and can also be smaller than $2 M_{\odot}$ prior to the explosion by mass loss.

The mass fraction of $\mathrm{O}$ in the O-rich layer is $\sim 0.8$. Other abundant elements in this layer are $\mathrm{Ne}, \mathrm{Mg}$, and $\mathrm{C}$, with mass fractions of order 0.1. These are almost irrespective of the evolutionary models. The He mass fraction in the He-rich layer is $\sim 0.9$. The second most abundant element in this layer is $\mathrm{C}$, with a mass fraction of $\sim 0.03$, but this is rather uncertain $(\S 3)$. Oxygen is also produced in the He-rich layer, but the mass fraction of $\mathrm{O}$ is only $\sim 0.01$.

\subsection{Hydrodynamics 85 Nucleosynthesis}

The hydrodynamics of the SN explosion and explosive nucleosynthesis are calculated for the five progenitor models. The hydrodynamic calculations are performed by a spherical Lagrangian hydrodynamic code with the piecewise parabolic method (PPM; Colella \& Woodward 1984). The code includes nuclear energy production from the $\alpha$ network. The equation of state includes gas, radiation, $\mathrm{e}^{-}-\mathrm{e}^{+}$pairs, Coulomb interaction between ions and electrons and phase transition (Nomoto 1982; Nomoto \& Hashimoto 1988). The explosion is initiated by increas-

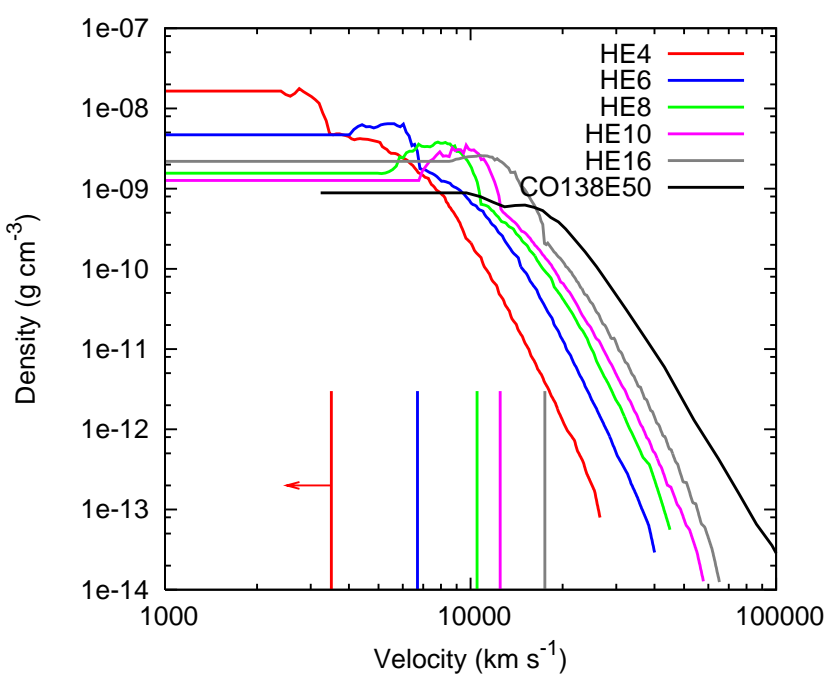

FiG. 2.- Density profile of the explosion models at one day after the explosion. Red (HE4), blue (HE6), green (HE8), magenta (HE10), and gray (HE16) lines show the models for SN 2008D (see Table 1). The black line shows the $\mathrm{C}+\mathrm{O}$ star explosion model used for SN 1998bw in Nakamura et al. (2001a). The vertical lines show the velocity at the bottom of the He layer in each model (with the same colors).

ing the temperatures at a few meshes below the mass cut (see below), i.e., a thermal bomb.

The SN ejecta become homologous at $\sim 1000 \mathrm{~s}$ after the explosion. After the hydrodynamic calculations, nucleosynthesis is calculated for each model as a postprocessing (Hix \& Thielemann 1996, 1999). The reaction network includes 280 isotopes up to ${ }^{79} \mathrm{Br}$. The results of the nucleosynthesis depends on the progenitor mass and the kinetic energy of the explosion. The kinetic energies in the five models are determined to explain the observed LC $(\S 3)$.

The explosion models are summarized in Table 1 . The mass cut $\left(M_{\text {cut }}\right)$ is defined after the nucleosynthesis calculation to eject the optimal amount of ${ }^{56} \mathrm{Ni}$ to power the LC. Figure 2 shows the density structure of the explosion models at one day after the initiation of the explosion. The "bump" in the density profile is caused by the reverse shock generated at the boundary of the $\mathrm{C}+\mathrm{O} / \mathrm{He}$ layers.

The vertical lines in Figure 2 show the velocity at the bottom of the He layer after the expansion of SN ejecta become homologous $\left(v_{\mathrm{He}}\right.$, Table 1$)$. Since strong mixing is expected in less massive stars $\left(M_{\alpha} \lesssim 4 M_{\odot}\right.$, Hachisu et al. 1991), the value of $v_{\mathrm{He}}$ in model HE4 is the upper limit of the inner velocity of the He-rich layer. If the mass of the He layer prior to the explosion is larger (smaller) than $\sim 2 M_{\odot}$ as in our model set, $v_{\mathrm{He}}$ can be lower (higher).

\section{BOLOMETRIC LIGHT CURVE}

The pseudo-bolometric (UBVRIJHK) LC was constructed by Minezaki et al. (in preparation, see Appendix A) compiling optical data taken by the MAGNUM telescope (Yoshii 2002; Yoshii, Kobayashi \& Minezaki 2003), the Himalayan Chandra Telescope, and Swift UVOT (U-band, Soderberg et al. 2008), and also NIR data taken by the MAGNUM telescope. The first part of the LC ( $t \lesssim 4$ days) seems to be related to the 

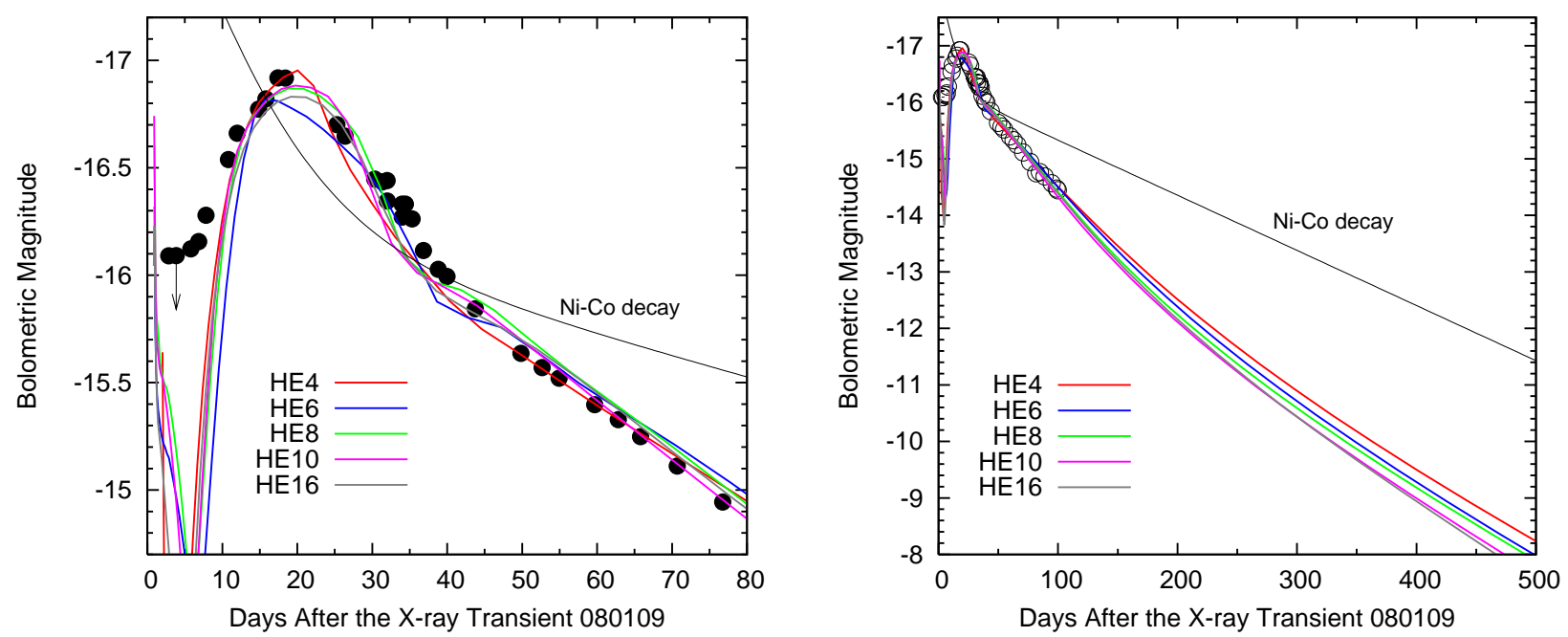

Fig. 3.- Pseudo-bolometric (UBVRIJHK) LC of SN 2008D (Minezaki et al. in preparation) compared with the results of LC calculations with the models HE4 (red), HE6(blue), HE8 (green), HE10 (magenta) and HE16 (gray). The pseudo-bolometric LC is shown in filled (left) and open (right) circles. The thin black line shows the decay energy from ${ }^{56} \mathrm{Ni}$ and ${ }^{56} \mathrm{Co}\left[M\left({ }^{56} \mathrm{Ni}\right)=0.07 M_{\odot}\right]$. The bolometric magnitude at $t \sim 4$ days after the X-ray transient is brighter by $\sim 0.25$ mag than that shown by other papers (Soderberg et al. 2008; Malesani et al. 2009; Modjaz et al. 2008b; Mazzali et al. 2008), which is shown by the thin arrow in the left panel (see Appendix A).

X-ray transient or the subsequent tail (Soderberg et al. 2008; Chevalier \& Fransson 2008) while the later part ( $t \gtrsim 4$ days) is the SN component, powered by the decay of ${ }^{56} \mathrm{Ni}$ and ${ }^{56} \mathrm{Co}$.

The first part of the $\mathrm{LC}$ depends on the progenitor radius and radiation-hydrodynamics at outer layers, as well as $M_{\mathrm{ej}}$ and $E_{\mathrm{K}}$. To determine the global properties of the SN ejecta, we focus on the second, principal part, which depends on $M_{\mathrm{ej}}, E_{\mathrm{K}}$ and the amount of ejected ${ }^{56} \mathrm{Ni}$ mass $\left[M\left({ }^{56} \mathrm{Ni}\right)\right]$. The progenitor radius is discussed in $\S 5.2$.

The LCs are calculated for the five explosion models presented in $\S 2$ (see Table 1). Our LTE, time-dependent radiative transfer code (Iwamoto et al. 2000) solves the Saha equation to obtain the ionization structure. Using the calculated electron density, the Rossland mean opacity is calculated approximately by the empirical relation to the electron scattering opacity derived from the TOPS database (Magee et al. 1995, Deng et al. 2005). For the initial temperature structure of the SN ejecta, we use results of adiabatic hydrodynamic calculations at one day after the explosion. The hydrodynamics and the radiative transfer are not coupled.

Asphericity of the ejecta of SN 2008D is suggested by the emission line profile in the spectrum at $t=109$ days (Modjaz et al. 2008b). To include the possible effect of aspherical explosion, we modify the distribution of ${ }^{56} \mathrm{Ni}$ from that derived from nucleosynthetic calculation. In hydrodynamic/nucleosynthetic calculations of aspherical explosion, more ${ }^{56} \mathrm{Ni}$ is mixed to the surface in the more aspherical cases (see e.g., Maeda et al. 2006, Tominaga 2009). A constant mass fraction of ${ }^{56} \mathrm{Ni}$ is assumed below $v_{\mathrm{Ni}}$, the outer boundary of ${ }^{56} \mathrm{Ni}$ distribution in velocity. The value of $v_{\mathrm{Ni}}$ is determined so as to explain the rising part of the LC. The estimated $v_{\mathrm{Ni}}$ is listed in Table 1 . The resultant mass fraction of ${ }^{56} \mathrm{Ni}$ is from 0.03 (HE4) to 0.01 (HE16).

Figure 3 shows the calculated LCs compared with the observed LC. The model LCs of HE8, HE10, and HE16 reproduce the observed LC around the peak very well. The LCs of HE4 and HE6 tend to be narrower than the observations. At a later phase, the five LCs are all in good agreement with the observations. The steep decline in the calculated LCs at $t \lesssim 4$ days could be a relic of the shock-heated envelope, and radiation-hydrodynamics calculations are required to study this part.

HE4 and HE6 need some enhancement of $\mathrm{C}$ in the He layer to reproduce the observed LC near the peak more nicely. The $\mathrm{C}$-abundance in the He layer is poorly known because of the uncertainties involved in the $\mathrm{C}$ production by convective $3 \alpha$-reaction in progenitor models and those in the Rayleigh-Taylor instability at the $\mathrm{He} / \mathrm{C}+\mathrm{O}$ interface during explosions, which tends to be stronger for lower mass He stars (Hachisu et al. 1991). In view of these uncertainties, we include HE4 and HE6 in the further spectral analysis, rather than excluding them from the possible models.

The timescale around the peak depends on both $M_{\mathrm{ej}}$ and $E_{\mathrm{K}}$ as $\propto \kappa^{1 / 2} M_{\mathrm{ej}}^{3 / 4} E_{\mathrm{K}}^{-1 / 4}$, where $\kappa$ is the optical opacity (Arnett 1982). Thus, for each model, a kinetic energy can be specified so as to reproduce the observed timescale. The derived set of ejecta parameters are $\left(M_{\mathrm{ej}} / M_{\odot}, E_{\mathrm{K}} / 10^{51} \mathrm{erg}\right)=(2.7,1.1),(4.4,3.7),(6.2$, $8.4),(7.7,13.0)$ and $(12.4,26.5)$ for the case of HE4, HE6, HE8, HE10 and HE16, respectively. The ejected ${ }^{56} \mathrm{Ni}$ mass is $\sim 0.07 M_{\odot}$ in all models.

The model with $M_{\mathrm{ej}}=3-5 M_{\odot}$ and $E_{\mathrm{K}}=2-4 \times 10^{51}$ erg suggested by Soderberg et al. (2008) is close to our HE6 model, while the model of Mazzali et al. (2008), with $M_{\mathrm{ej}}=7 M_{\odot}$ and $E_{\mathrm{K}}=6 \times 10^{51} \mathrm{erg}$ is close to our HE8 model. Model HE4 has the canonical explosion energy of core-collapse SNe (i.e., $\sim 10^{51}$ erg) while HE10 and HE16 have the explosion energy of hypernovae ( $>$ $\left.10^{52} \mathrm{erg}\right)$.

In all five models, the late evolution of the $\mathrm{LC}(t>$ 


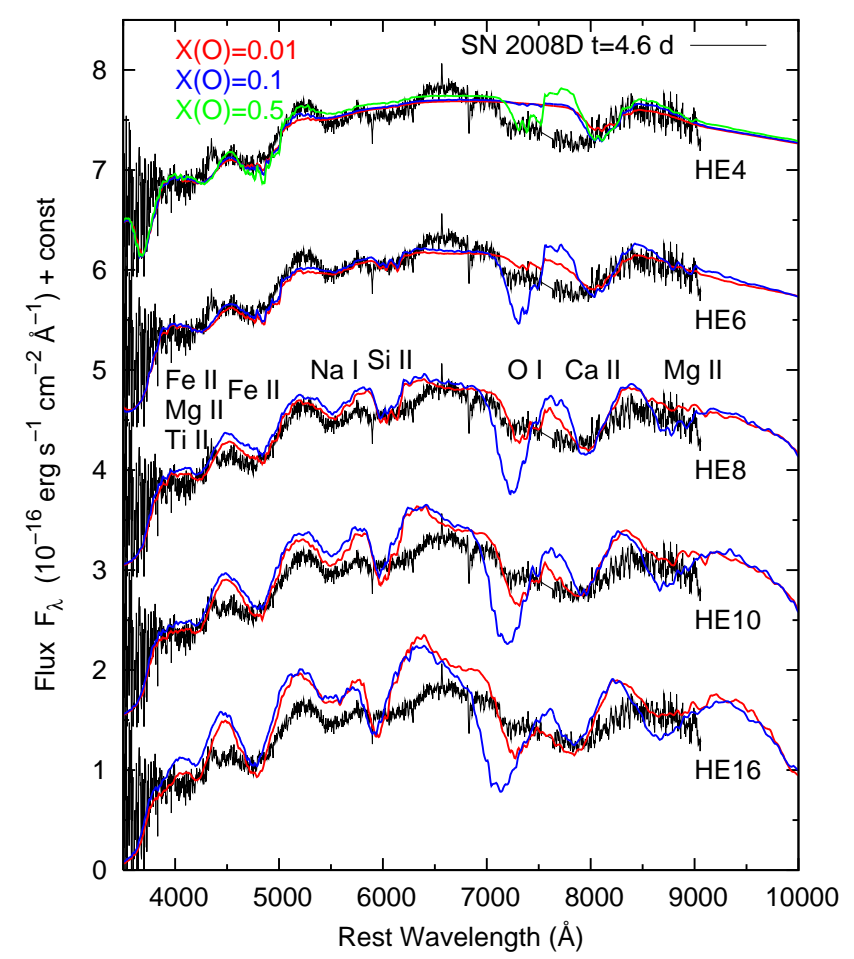

FIG. 4.- Spectrum of SN 2008D at $t=4.6$ days from the X-ray transient (black line, Mazzali et al. 2008) compared with synthetic spectra (color lines). The spectra are shifted by $6.0,4.5,3.0,1.5$, 0.0 from top to bottom. The model spectra are reddened with $E(B-V)=0.65 \mathrm{mag}$. From top to bottom, the synthetic spectra calculated with HE4, HE6, HE8, HE10, and HE16 are shown. The red, blue and green lines show the synthetic spectra with oxygen mass fraction $X(\mathrm{O})=0.01,0.1$, and 0.5 , respectively. Since the synthetic spectra with $X(\mathrm{O})=0.1$ for more massive models than HE4 already show too strong $\mathrm{O}$ I line, the spectra with $X(\mathrm{O})=$ 0.5 are not shown for these models.

200 days) is not very different, with a decline rate of $\sim 0.015$ mag day ${ }^{-1}$ (right panel of Fig. 3). This decline is faster than the ${ }^{56}$ Co decay rate $\left(0.01 \mathrm{mag}\right.$ day $^{-1}$, thin black line in Fig. 3) because some $\gamma$-rays escape without depositing energy in the SN ejecta at such late epochs. These models predict that the optical magnitude of SN $2008 \mathrm{D}$ is $\sim-10.5 \mathrm{mag}$ (observed magnitude $\sim 23.8$ with no bolometric correction) in 2008 October, i.e., $\sim 300$ days after the explosion, when the SN can be observed again, and $\sim-9.6 \mathrm{mag}(\sim 24.7 \mathrm{mag})$ at one year after the explosion (if dust does not form in the ejecta).

\section{OPTICAL SPECTRA}

In this section, the five models are tested against the observed spectra. Optical spectra have been shown by Soderberg et al. (2008), Malesani et al. (2009), Modjaz et al. (2008b) and Mazzali et al. (2008). We use the data set presented by Mazzali et al. (2008). The spectral sequence can be divided into three parts. At the earliest epochs ( $t \lesssim 4$ days), the spectra are almost featureless 16. This is probably the result of shock heating (the first part of the LC). At $4 \lesssim t \lesssim 10$ days, the spectra show

16 Two absorption features are identified around $4000 \AA$ in the spectra at $t \sim 2$ days $(t=1.77$ days, Malesani et al. $2009 ; t=1.84$ days, Modjaz et al. 2008b), while they are not seen in the spectra at $t=1.54$ and 2.49 days presented by Mazzali et al. (2008). These absorptions might be due to more highly-ionized ions, such as $\mathrm{C}$ III, broad-line features. Around and after maximum $(t \gtrsim 10$ days), the spectrum shows strong He features as in Type Ib SNe. The velocity of the He lines is $\sim 9000-10,000$ $\mathrm{km} \mathrm{s}^{-1}(\S 4.3)$. We present spectral modelling at the SN dominated phase, i.e., $t \gtrsim 4$ days.

For spectral modelling, we use the one-dimensional Monte Carlo spectrum synthesis code (Mazzali \& Lucy 1993). The code assumes a spherically symmetric, sharply defined photosphere. Electron and line scattering are taken into account. For line scattering, the effect of line branching is included (Lucy 1999; Mazzali 2000). The ionization structure is calculated with modified nebular approximation as in Mazzali \& Lucy (1993, see also Abbott \& Lucy 1985). Although it is known that the non-thermal excitation is important for the He lines (Lucy 1991), non-thermal processes are not included in our analysis. Thus, we do not aim to obtain a good fit of the He lines.

To determine the temperature structure, many photon packets are first traced above the photosphere with an assumed temperature structure. The Monte Carlo ray tracing gives the flux at each mesh and the temperature structure is then updated using the flux. This procedure is repeated until the temperature converges. Finally a model spectrum is obtained using a formal integral (Lucy 1999).

The input parameters of the code are emergent luminosity $(L)$, the position of the photosphere in velocity (photospheric velocity, $v_{\mathrm{ph}}$ ), and element abundances (mass fractions) above the photosphere (i.e., in the SN atmosphere). Note that $L$ and $v_{\text {ph }}$ do not depend much on the model parameters such as $M_{\mathrm{ej}}$ and $E_{\mathrm{K}}$. They are constrained by the absolute flux of the spectrum and the line velocities, respectively (and also by the relation of $L \propto v_{\mathrm{ph}}^{2} t^{2} T_{\mathrm{eff}}^{4}$, where $T_{\mathrm{eff}}$ is the effective temperature of the spectrum).

With the estimated luminosity and photospheric velocity, mass fractions of elements are optimized. For simplicity, homogeneous abundances are assumed above the photosphere without using the results of nucleosynthetic calculations. We compare the derived abundances with those by nucleosynthetic calculations for the progenitor models. The goodness of the fit is judged by eyes because of the complex dependences of the parameters and the difficulty in obtaining the perfect fit of the overall spectrum.

\subsection{Broad-Line Spectrum: At $t=4.6$ Days}

We first perform model calculations for the spectrum at $t=4.6$ days (Fig. 4). The spectrum shows broad-line features.

\subsubsection{Intermediate Mass Model HE8}

We use model HE8, the middle of our model sequence, as a fiducial case. A good agreement with the observed spectrum is obtained with $v_{\mathrm{ph}}=18,500 \mathrm{~km} \mathrm{~s}^{-1}$ and $\log$ $L\left(\mathrm{erg} \mathrm{s}^{-1}\right)=41.7$. Since this velocity is higher than the

N III. and O III (Modjaz et al. 2008b; Quimby et al. 2007). We have investigated these lines by the Monte Carlo spectrum synthesis code, but we don't find a large contribution of these ions because ionizations by the photospheric radiation only is not enough for the strong contributions of such ions, as noted by Modjaz et al. (2008b). 
He line velocities observed later phases $(\sim 9000-10,000$ $\mathrm{km} \mathrm{s}^{-1}$ ), the photosphere at this epoch is expected to be located in the He-rich layer.

Figure 4 shows a comparison of the observed and synthetic spectrum. The spectrum has P-Cygni profiles of $\mathrm{O}$ I, Na I, Ca II, Ti II, Cr II, and Fe II lines. The line at $6000 \AA$ is identified as Si II. The contribution of the high velocity $\mathrm{H} \alpha$ is quite small, which is discussed in Appendix B. The spectrum at wavelengths bluer than $5500 \AA$ is dominated by Ti II, Cr II, and Fe II lines. Given the uncertainty in the metal abundances in outer layers, reflecting the uncertainty of the explosion mechanism or the degree of mixing, these features can be fitted using the optimal value of the metal abundances.

In contrast to the heavy, synthesized elements, the oxygen abundance cannot be totally parameterized because the majority of oxygen is synthesized during the evolution of the progenitor star. The red and blue line shows synthetic spectra with oxygen abundance $X(\mathrm{O})=0.01$ and 0.1 , respectively. In these models, the abundance of $\mathrm{He}$ is $X(\mathrm{He}) \sim 0.8$ and 0.7 , respectively. The spectrum with $X(\mathrm{O})=0.01$ (red) gives a good match with the observed O I $\lambda 7774$ line around $7400 \AA$, while the O I line in the model spectrum with $X(\mathrm{O})=0.1$ (blue) is too strong. Since the oxygen abundance in the He-rich layer is of the order of $10^{-2}$ almost irrespective of evolutionary models, this is consistent with the fact that the photosphere is located in the He-rich layer.

In the observed spectrum, the O I and Ca II IR triplet are blended at $7000-8500 \AA$ while they are separated in the synthetic spectra. This is caused by the insufficient Ca II absorption in the model at the very high velocity layer with $v \sim 0.1 c$. The ejecta mass at $v>0.1 c$ in HE8 is $0.04 M_{\odot}$ (Table 1$)$, which is consistent with that in the model presented by Mazzali et al. (2008). Note that the mass at $v>0.1 c$ is much smaller than in the model for SN 1998bw $\left(\sim 1.5 M_{\odot}\right.$, CO138E50 in Nakamura et al. 2001a, see also Fig. 2).

\subsubsection{Massive Models HE10 and HE16}

Next, we use the more massive models. For model HE10, the $\mathrm{O}$ I line in the model with $X(\mathrm{O})=0.01$ (red) seems to be strong, but we can obtain a good fit with slightly smaller oxygen abundance. For model HE16, the strength of the $\mathrm{O}$ I line with $X(\mathrm{O})=0.01$ (red) is similar to that in HE10. In these massive models, the $\mathrm{O}$ I feature is too strong in the model spectra with $X(\mathrm{O})=0.1$ (blue). This is consistent with the fact that the photosphere $(v=$ $18,500 \mathrm{~km} \mathrm{~s}^{-1}$ ) is located in the He-rich layer.

\subsubsection{Less Massive Models HE6 and HE4}

Finally, we use less massive models. For the less massive model HE6, $X(\mathrm{O})=0.01$ gives a reasonable fit to the $\mathrm{O}$ I line. In contrast, $X(\mathrm{O})=0.1$ yields too strong a line. This is a similar behavior to the more massive models, and implies that the photosphere is located in the He-rich layer.

For HE4, the synthetic spectra with $X(\mathrm{O})=0.01$ and 0.1 do not give a strong enough $\mathrm{O}$ I absorption (red and blue lines). To explain the observed absorption, $X(\mathrm{O})=0.5$ is required (green line) because of the low density at the outer layer of HE4 (Fig. 2). This requires that the layer at $v=18,500 \mathrm{~km} \mathrm{~s}^{-1}$ should already be

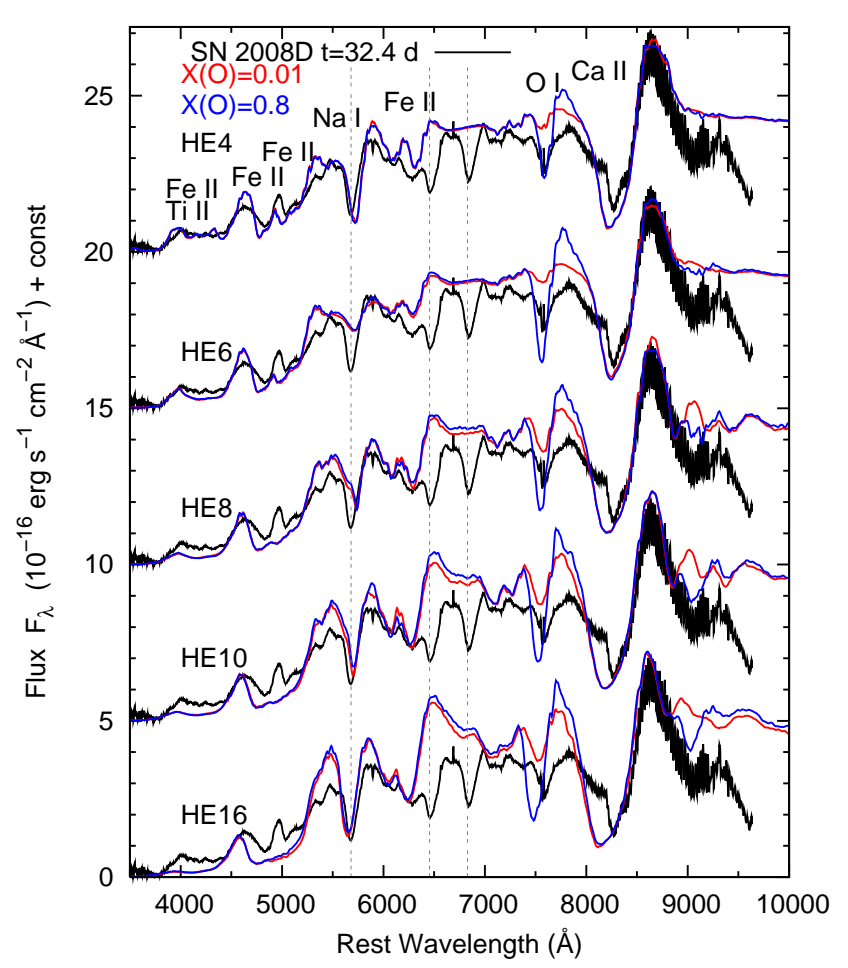

Fig. 5.- Spectrum of SN 2008D at $t=32.4$ days from the X-ray transient (black line, Mazzali et al. 2008) compared with synthetic spectra (color lines). The spectra are shifted by 20.0, 15.0, 10.0, $5.0,0.0$ from top to bottom. The model spectra are reddened with $E(B-V)=0.65 \mathrm{mag}$. From top to bottom, the synthetic spectra calculated with HE4, HE6, HE8, HE10, and HE16 are shown. The red and blue lines show the synthetic spectra with an oxygen mass fraction of $X(\mathrm{O})=0.01$ and 0.8 , respectively. Dashed, vertical lines show the position of the He lines (He I 5876, 6678, 7065) blueshifted with $v=10,000 \mathrm{~km} \mathrm{~s}^{-1}$. Note that the He lines are not treated in the code.

O-rich, which is clearly inconsistent with the observed He line velocity $\left(v \sim 9000-10,000 \mathrm{~km} \mathrm{~s}^{-1}\right)$. Therefore, HE4 is not likely to be a viable model for SN 2008D.

\subsection{Type Ib Spectrum: At $t=32.4$ Days}

At $t=32.4$ days, the observed spectrum shows typical Type Ib features. The overall features are fitted well with $v_{\mathrm{ph}}=7500 \mathrm{~km} \mathrm{~s}^{-1}$ and $\log L\left(\mathrm{erg} \mathrm{s}^{-1}\right)=42.1$ (Fig. 5). The observed Fe lines at $4500-5000 \AA$ are too narrow to be reproduced by the massive models (HE8, HE10 and HE16). This is caused by our crude assumption of a homogeneous abundance distribution, which is not appropriate for synthesized elements such as Fe in the inner layer. The spectra might be improved using a stratified abundance distribution or non-spherical models (Tanaka et al. 2007).

\subsubsection{Intermediate Mass Model HE8}

The model spectrum with $X(\mathrm{O})=0.01$, as assumed for the spectrum at $\mathrm{t}=4.6$ days, is shown in red line. The synthetic $\mathrm{O}$ I line at $7500 \AA$ is slightly weaker than the observation. A value $X(\mathrm{O})=0.8$ yields a reasonably strong $\mathrm{O}$ I line (blue), which implies that the photosphere $\left(v=7500 \mathrm{~km} \mathrm{~s}^{-1}\right)$ is not located in the He-rich layer, consistent with $v_{\mathrm{He}}=10500 \mathrm{~km} \mathrm{~s}^{-1}$ (velocity at the bottom of the He layer) of HE8. 


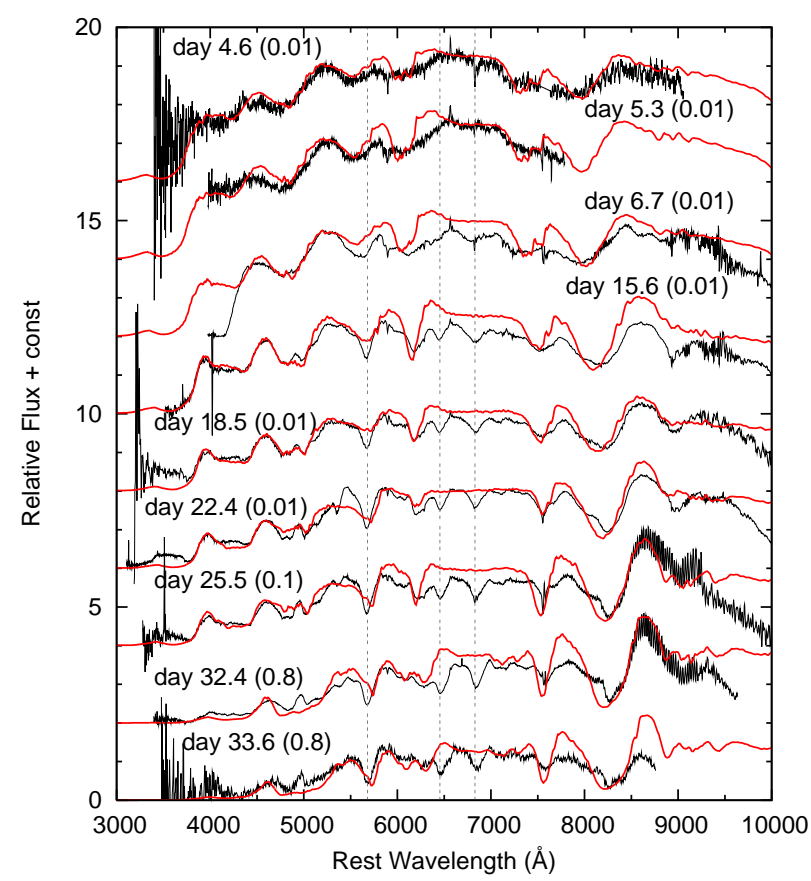

FIG. 6.- Spectral evolution of SN 2008D (black, Mazzali et al. 2008) compared with the sequence of the synthetic spectra computed with HE8 (red). The epoch in the figure shows the days from the X-ray transient. The values in the parenthesis shows the mass fraction of oxygen assumed in the calculation. The spectra are shifted by $16.0,14.0,12.0,10.0,8.0,6.0,4.0,2.0,0.0$ from top to bottom. The model spectra are reddened with $E(B-V)=0.65$ mag. Dashed, vertical lines show the position of the He lines (He I $5876,6678,7065)$ blueshifted with $v=10,000 \mathrm{~km} \mathrm{~s}^{-1}$. Note that the He lines are not treated in the code.

\subsubsection{Massive Models HE10 and HE16}

The synthetic spectra calculated using HE10 and HE16 show the stronger $\mathrm{O}$ I line than HE8. The spectrum with $X(\mathrm{O})=0.01$ (red) gives a slightly weaker $\mathrm{O}$ I line than in the observation, while the spectrum with $X(\mathrm{O})=0.8$ (blue) yields a sufficiently strong line. Although the synthetic $\mathrm{O}$ I line with $X(\mathrm{O})=0.8$ is too strong especially at high velocity (i.e., at bluer wavelength), this is caused by the assumption of the homogeneous abundance distribution. Thus, near the photosphere, a high mass fraction of $\mathrm{O}$ is preferred. This is consistent with the high $v_{\mathrm{He}}$ of these models $(12,500$ and 17, 500 for HE10 and HE16, respectively).

However, the observed He line velocities $(v \sim 9000$ $10,000 \mathrm{~km} \mathrm{~s}^{-1}$ ) suggest that the layer at $v \sim 10,000$ $\mathrm{km} \mathrm{s}^{-1}$ is still He-rich. This is inconsistent with the high $v_{\mathrm{He}}$ in HE10 and HE16, requiring that the layers at $v \sim 10,000 \mathrm{~km} \mathrm{~s}^{-1}$ be O-rich.

\subsubsection{Less Massive Models HE6 and HE4}

The synthetic spectra using HE6 also have similar trend with those of HE8. The spectrum with $X(\mathrm{O})=0.8$ (blue) gives a reasonable fit to the $\mathrm{O}$ i line. Although the low velocity at the bottom of the He layer in HE6 $\left(v_{\mathrm{He}}=6700 \mathrm{~km} \mathrm{~s}^{-1}\right)$ suggests that the photosphere at this epoch $\left(v=7500 \mathrm{~km} \mathrm{~s}^{-1}\right)$ is still in the He layer, this small difference is within the uncertainty of $v_{\mathrm{He}}$ caused by the variation of the He layer mass depending on evolutionary models.

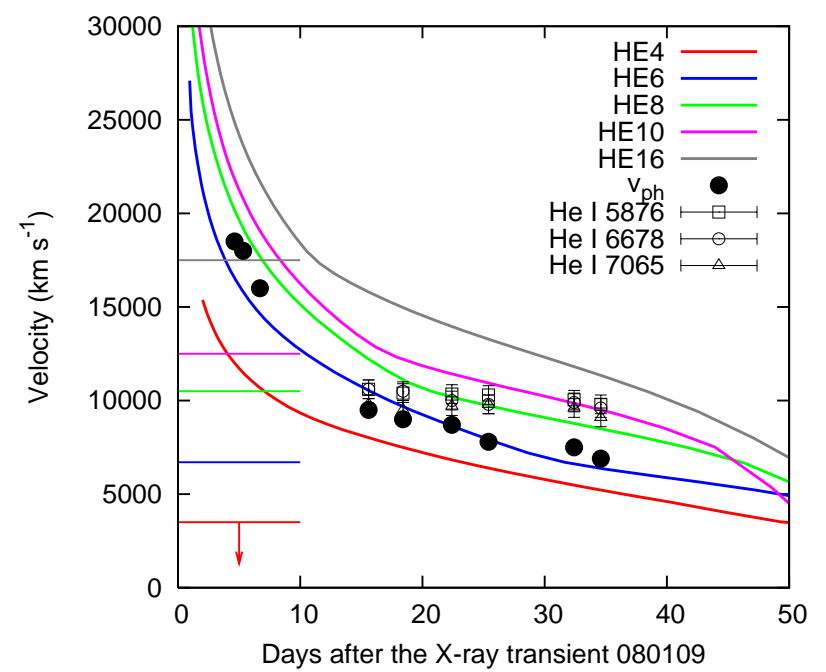

FIG. 7.- Time evolution of photospheric velocity calculated by the LC code (color lines) and that derived from the spectral modelling (filled circles). Open black symbols show the line velocity of He lines. The horizontal lines show $v_{\mathrm{He}}$ for each model (Table $1)$. Note that $v_{\mathrm{He}}$ can be varied depending on evolutionary models with different masses of the He layer.

For HE4, the $\mathrm{O}$ I absorption is reproduced with $X(\mathrm{O})=$ 0.8 . However, the very low $v_{\mathrm{He}}$ of HE4 $\left(<3500 \mathrm{~km} \mathrm{~s}^{-1}\right)$ is not consistent with the fact that the spectrum model requires the $\mathrm{O}$-dominated photosphere at $v=7500 \mathrm{~km} \mathrm{~s}^{-1}$.

\subsection{Velocity Evolution}

Using the fiducial model HE8, we calculate the spectral evolution (Fig. 6). The values in the parenthesis is the oxygen mass fraction adopted in the fitting. We find that a higher oxygen mass fraction is preferred for the later spectra. Although a homogeneous mass fraction is assumed in the calculation, the photospheric position seems to transit from the He-rich layer to the O-rich layer around $t=25.5$ days. Thus, the boundary between the He-rich and O-rich layers is located near $v=7800$ $\mathrm{km} \mathrm{s}^{-1}$.

Figure 7 shows the photospheric velocities derived from the spectral modelling (filled black circles), which does not depend much on the model parameters. In Figure 7, the photospheric velocities obtained from the synthetic LCs (§3) are also shown (solid lines). The photospheric velocities for HE6 and HE8 are close to the values derived from spectral modelling. However, it should be noted that the photospheric velocities obtained from the LC models are only approximate because the LC model assumes LTE and does not fully take into account the contribution of the line opacity. Thus, uncertainty of a few thousand $\mathrm{km} \mathrm{s}^{-1}$ is expected. Nevertheless, the decreasing trend of the photospheric velocity derived from the spectral modelling is reproduced by our LC calculations because (1) we use the hydrodynamic models (Fig. 2 ) having a decreasing density structure toward the outer layers and (2) we solve the ionization in the ejecta, and thus, the opacity is time-dependent.

In Figure 7, the Doppler velocities of three He I lines measured at the absorption minimum (open symbols) are also shown. Malesani et al. (2009) and Modjaz et al. (2008b) show the subsequent spectral evolution, and the He line velocity declines slowly to $v \sim 9000 \mathrm{~km} \mathrm{~s}^{-1}$. 

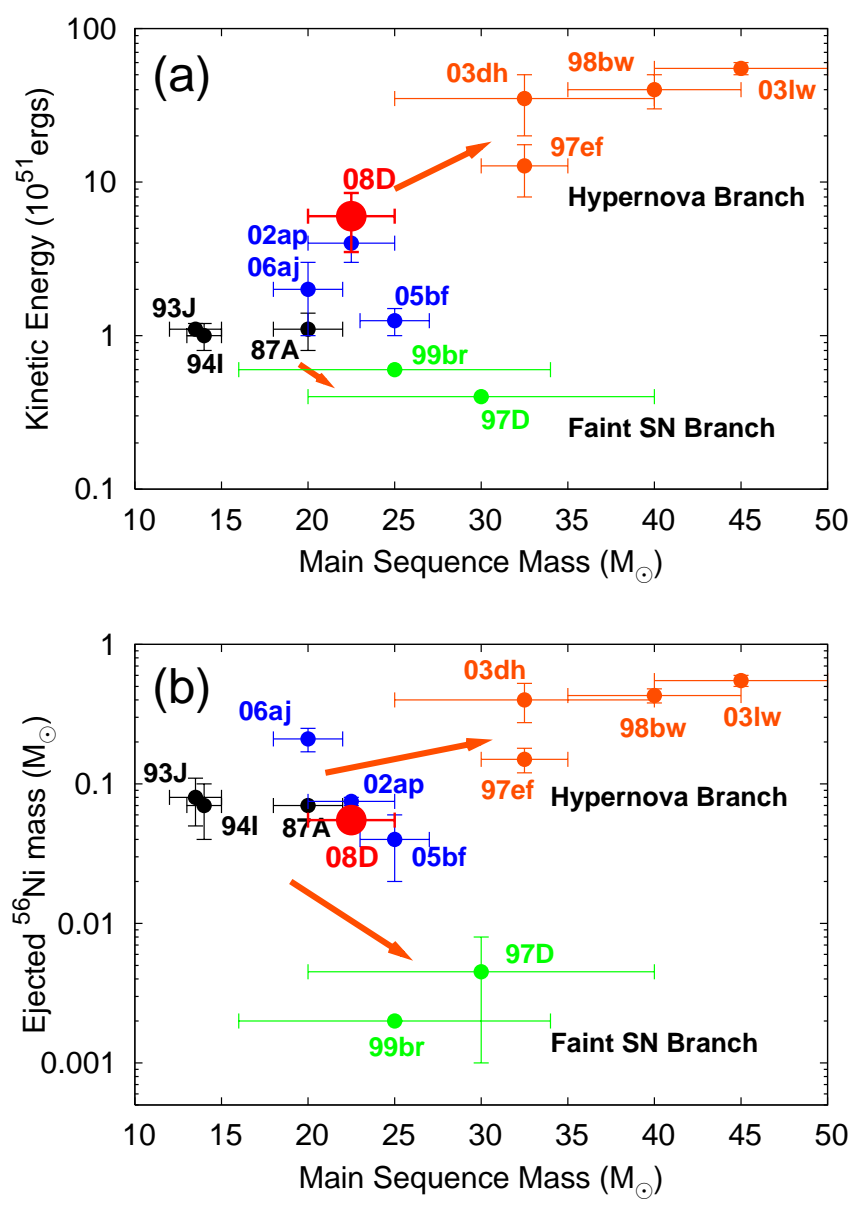

FIG. 8.- Kinetic energy of the explosion (upper) and the ejected ${ }^{56} \mathrm{Ni}$ mass as a function of the estimated main-sequence mass of the progenitors for several core-collapse SNe. The parameters are listed in Table 2 with references. The progenitor mass of $\mathrm{SNe}$ shown in the figure is estimated based on the $M_{\mathrm{MS}}-M_{\alpha}$ relation by Sugimoto \& Nomoto (1980; used in Nomoto \& Hashimoto 1988) as in this paper.

The horizontal lines in Figure 7 mark the velocity at the bottom of the He layer for the five models $\left(v_{\mathrm{He}}\right.$, see Table 1). In HE10 and HE16, $v_{\mathrm{He}}$ is too high compared with the observed velocities (\$4.2.2). Also in HE8, it may be higher than the minimum of the observed He line velocity $\left(v \sim 9000 \mathrm{~km} \mathrm{~s}^{-1}\right)$. The lower $v_{\mathrm{He}}$ in HE4 and HE6 cannot be excluded from the observed line velocities. But the spectral modelling shows that the layer at $v \sim$ $7500 \mathrm{~km} \mathrm{~s}^{-1}$ is not He-rich (§4.2). This is inconsistent with the very low $v_{\mathrm{He}}$ in $\mathrm{HE} 4$. It must be cautioned that $v_{\mathrm{He}}$ is affected by the mass of the He layer (i.e., by the choice of evolutionary models).

\section{DISCUSSION}

\subsection{Optimal Model for $S N$ 2008D}

For the five He star progenitor models, we calculate hydrodynamics of the explosions and explosive nucleosynthesis. To reproduce the observed LC, we obtain the possible set of the mass and kinetic energy of the ejecta: $\left(M_{\mathrm{ej}} / M_{\odot}, E_{\mathrm{K}} / 10^{51} \mathrm{erg}\right)=(2.7,1.1),(4.4,3.7),(6.2,8.4)$, $(7.7,13.0)$ and $(12.4,26.5)$ for HE4, HE6, HE8, HE10 and HE16, respectively. These five models are tested against the optical spectra.

Model HE4 has many difficulties in reproducing the observed spectra. At early epochs, the calculated O I line is too weak because of the too small oxygen mass in the He-rich layer. At later epoch, the model spectrum suggests that the photospheric layer at $v=7500$ $\mathrm{km} \mathrm{s}^{-1}$ is O-rich, which is not consistent with the explosion model that has He-rich or He-O mixed layers at $v \gtrsim 3000 \mathrm{~km} \mathrm{~s}^{-1}$.

Model HE6 can reproduce the observed spectra well. The evolution of the photospheric velocity calculated with HE6 is in reasonable agreement with the velocities derived from the spectral modelling (Fig. 7). The spectral model at $t=32.4$ suggests that the layer at $v \sim 7500$ $\mathrm{km} \mathrm{s}^{-1}$ is not the He-rich layer, while the slightly lower $v_{\mathrm{He}}$ of HE6 $\left(6700 \mathrm{~km} \mathrm{~s}^{-1}\right)$ implies that the photosphere at this epoch is He-rich.

Model HE8 is reasonably consistent with all the aspects studied in this paper At all epochs, the optical spectra can be explained with a reasonable abundance distribution, and the calculated photospheric velocities are consistent with those derived from spectrum synthesis. However, the velocity at the bottom of the He layer $\left(v_{\mathrm{He}}\right)$ in $\mathrm{HE} 8$ is slightly higher than the observed He line velocities.

Model HE10 and HE16 reproduce the early and later spectra reasonably well. However, these models predict too high photospheric velocity (Fig. 7). In addition, the velocities at the bottom the He layer $\left(v_{\mathrm{He}}=12,500\right.$ and $17,500 \mathrm{~km} \mathrm{~s}^{-1}$ for HE10 and HE16, respectively), are not consistent with the observed line velocity $(v \sim 9000$ - $10,000 \mathrm{~km} \mathrm{~s}^{-1}$ ).

In summary HE4, HE10 and HE16 are not consistent with SN 2008D. Both HE6 and HE8 have a small inconsistency related to the boundary between the He-rich and O-rich layers. It seems that a model between HE6 and HE8 may be preferable. However, since there is uncertainty in $v_{\mathrm{He}}$ in our model set, depending on the mass of the He layers, we include both HE6 and HE8 as possible models.

We conclude that the progenitor star of SN 2008D has a He core mass $M_{\alpha}=6-8 M_{\odot}$ prior to the explosion. This corresponds to a main-sequence mass of $M_{\mathrm{MS}}=$ $20-25 M_{\odot}$ under the $M_{\mathrm{MS}}-M_{\alpha}$ relation by Sugimoto \& Nomoto (1980; used in Nomoto \& Hashimoto 1988). We find that SN 2008D is an explosion with $M_{\mathrm{ej}}=5.3 \pm$ $1.0 M_{\odot}$ and $E_{\mathrm{K}}=6.0 \pm 2.5 \times 10^{51} \mathrm{erg}$. The mass of the central remnant is $1.6-1.8 M_{\odot}$, which is near the boundary mass between the neutron star and the black hole. Note that the error bars only reflect the uncertainty of the LC and spectral modelling. Possible additional uncertainties of the parameters are discussed below.

Distance and reddening: since the distance to the host galaxy and the reddening toward the SN include some uncertainties, the ejected ${ }^{56} \mathrm{Ni}$ mass could also contain $\sim 20 \%$ uncertainties. However, $M_{\mathrm{ej}}$ and $M_{\text {cut }}$ are not affected because these values are much larger than the ejected ${ }^{56} \mathrm{Ni}$ mass. Thus, the estimated core mass and progenitor mass are not largely affected by the uncertainty of the distance and the reddening.

Asphericity of the explosion: possible effects on the estimate of $M_{\mathrm{ej}}$ and $E_{\mathrm{K}}$ from asphericity of the ejecta are of interest. These effects were studied for SN 1998bw 
Type Ib Supernova 2008D

TABLE 2

Parameters of Supernovae

\begin{tabular}{|c|c|c|c|c|c|}
\hline SN (Type) & $M_{\mathrm{ej}}^{\mathrm{a}}$ & $E_{\mathrm{K}}{ }^{\mathrm{b}}$ & $M\left({ }^{56} \mathrm{Ni}\right)^{\mathrm{c}}$ & $M_{\mathrm{MS}}{ }^{\mathrm{d}}$ & Refs. \\
\hline SN 1987A (II pec) & 14.7 & $1.1 \pm 0.3$ & 0.07 & $20 \pm 2$ & 1,2 \\
\hline SN 1993J (IIb) & $3.2 \pm 0.3$ & $1.1 \pm 0.1$ & $0.08 \pm 0.03$ & $13.5 \pm 1.5$ & 3 \\
\hline SN 1994I (Ic) & $1.05 \pm 0.15$ & $1.0 \pm 0.2$ & $0.07 \pm 0.03$ & $14 \pm 1$ & 4,5 \\
\hline SN 1997D (II) & $\sim 24$ & $\sim 0.4$ & $0.0045 \pm 0.0035$ & $30 \pm 10$ & 6 \\
\hline SN 1997ef (Ic) & $8.6 \pm 1$ & $12.75 \pm 4.75$ & $0.15 \pm 0.03$ & $32.5 \pm 2.5$ & 7,8 \\
\hline SN 1998bw (Ic) & $10.4 \pm 1$ & $40 \pm 10 \mathrm{e}^{\mathrm{s}}$ & $0.43 \pm 0.05$ & $40 \pm 5$ & 9,10 \\
\hline SN 1999br (II) & $\sim 14$ & $\sim 0.6$ & 0.002 & $25 \pm 9$ & 11 \\
\hline SN 2002ap (Ic) & $3.25 \pm 0.75$ & $4 \pm 1$ & $0.075 \pm 0.005$ & $22.5 \pm 2.5$ & 12 \\
\hline SN 2003dh (Ic) & $7 \pm 3$ & $35 \pm 15$ & $0.4 \pm 0.125$ & $32.5 \pm 7.5$ & 13,14 \\
\hline SN 2003lw (Ic) & $\sim 13$ & $55 \pm 5$ & $0.55 \pm 0.05$ & $45 \pm 5$ & 15 \\
\hline SN 2005bf (Ib pec) & $6.5 \pm 0.5$ & $1.25 \pm 0.25$ & $0.04 \pm 0.02^{f}$ & $25 \pm 2$ & 16,17 \\
\hline SN 2006aj (Ic) & $1.8 \pm 0.8$ & $2.0 \pm 1.0$ & $0.21 \pm 0.04$ & $20 \pm 2$ & 18 \\
\hline SN 2008D (Ib) & $5.3 \pm 1.0$ & $6.0 \pm 2.5$ & $0.07 \pm 0.005$ & $22.5 \pm 2.5$ & this work \\
\hline
\end{tabular}

REFERENCES. - (1) Shigeyama \& Nomoto (1990), (2) Blinnikov et al. (2000), (3) Shigeyama et al. (1994), (4) Iwamoto et al. (1994), (5) Sauer et al. (2006), (6) Turatto et al. (1998), (7) Iwamoto et al. (2000), (8) Mazzali, Iwamoto \& Nomoto (2000), (9) Nakamura et al. (2001a), (10) Iwamoto et al. (1998), (11) Zampieri et al. (2003), (12) Mazzali et al. (2002), (13) Mazzali et al. (2003), (14) Deng et al. (2005), (15) Mazzali et al. (2006a), (16) Tominaga et al. (2005), (17) Maeda et al. (2007), (18) Mazzali et al. (2006b)

aThe mass of the SN ejecta $\left(M_{\odot}\right)$

${ }^{\mathrm{b}}$ The kinetic energy of the SN ejecta $\left(10^{51} \mathrm{erg}\right)$

${ }^{\mathrm{c}}$ The mass of ejected ${ }^{56} \mathrm{Ni}\left(M_{\odot}\right)$

${ }^{\mathrm{d}}$ Estimated main-sequence mass $\left(M_{\odot}\right)$

${ }^{\mathrm{e}} E_{\mathrm{K}}=20 \times 10^{51} \mathrm{erg}$ is derived from the modelling with a multi-dimensional model (in the polar-viewed case, Maeda et al. 2006; Tanaka et al. 2007)

${ }^{\mathrm{f}}$ The mass of ${ }^{56} \mathrm{Ni}$ is derived from the late time observation (Maeda et al. 2007). The early observations suggest $M\left({ }^{56} \mathrm{Ni}\right)=0.3 M_{\odot}$ (Tominaga et al. 2005; Folatelli et al. 2006).

associated with GRB 980425 by Maeda et al. (2006) and Tanaka et al. (2007). They found that the kinetic energy can be smaller by a factor of $\lesssim 2$ in the on-axis case of highly aspherical explosion than in the spherical model. There is little effect in the off-axis case.

Modjaz et al. (2008b) presented the spectrum at $t=109$ days and suggested the asphericity of SN 2008D by the doubly-peaked emission profile of the $\mathrm{O}$ I line. Such a profile of the $\mathrm{O}$ I line has been interpreted as an off-axis line-of-sight in the axisymmetric explosion (Maeda et al. 2002; Mazzali et al. 2005; Maeda et al. 2008; Modjaz et al. 2008a). Thus, the estimate by the modelling under spherical symmetry may not be largely changed even for the aspherical models. The quantitative discussion should wait for the later spectra, the detailed modelling of the line profile, and determination of the degree of asphericity and the line-of-sight.

The effects of asphericity, especially aspherical mass ejection and fallback, are also important to determine the relation between the ejected ${ }^{56} \mathrm{Ni}$ mass and the remnant mass. The remnant mass in this paper is determined to eject the optimal amount of ${ }^{56} \mathrm{Ni}$ by one-dimensional hydrodynamic/nucleosynthetic calculations. However, since the remnant mass could be either larger or smaller depending on the asphericity and details of the explosion mechanism, the estimate by one-dimensional calculations is a reasonable approximation.

Possible presence of hydrogen: Soderberg et al. (2008) identified the high velocity $\mathrm{H} \alpha$ for the absorption line at $6150 \AA$. If the mass of the $\mathrm{H}$ layer is not negligible, it might affect the core mass, which we estimate by assuming non-existence of $\mathrm{H}$, i.e., a bare He core. However, we find a large mass of the $\mathrm{H}$ layer is inconsistent with the spectrum at $t=4.6$ days (Appendix B). The mass of the $\mathrm{H}$ layer is smaller than $5 \times 10^{-4} M_{\odot}$, and thus, there is no effect on the parameters.

Evolutionary models: the estimate of the mainsequence mass uses the approximate $M_{\mathrm{MS}}-M_{\alpha}$ relation by Sugimoto \& Nomoto (1980; Eq. 4.1), which is used in Nomoto \& Hashimoto (1988). The $M_{\mathrm{MS}}-M_{\alpha}$ relation of several evolutionary models are shown in Figure 1. The systematic differences in this relation for $M_{\mathrm{MS}} \lesssim 30 M_{\odot}\left(M_{\alpha} \lesssim 10 M_{\odot}\right)$ may stem from the differences in the treatment of convection, mass loss, rotation, and binary effects (§2.1). Thus, we should keep in mind that the main-sequence mass is subject to systematic uncertainties of $3-5 M_{\odot}$ (Fig. 1). Note that our estimate of the He core mass depends only on the estimates of $M_{\mathrm{ej}}$ and $E_{\mathrm{K}}$ from hydrodynamic/nucleosynthetic calculations, and thus, our determination of the He core mass is not affected by the variety of the evolutionary models.

\subsection{Comparison with Previous Works}

Soderberg et al. (2008) have estimated the parameters of the ejecta as $M_{\mathrm{ej}}=3-5 M_{\odot}$ and $E_{\mathrm{K}}=2-4 \times 10^{51}$ ergs, which are smaller than those derived in this paper. The difference seems to stem from their assumptions of the homogeneous sphere and time-independent opacity. These assumptions lead an almost time-independent photospheric velocity, which is not the case in SNe. Especially for SN 2008D, the very early spectra show the broad-line features, and the photospheric velocity at $t \sim 5$ days after the X-ray transient is almost twice as high as the velocity around maximum.

Adopting the cooling envelope model by Waxman et al. (2007) to the blackbody temperature and the radius at $t \lesssim 4$ days, Soderberg et al. (2008) estimated a progenitor radius to be $R_{*} \sim 1 R_{\odot}$ with $E(B-V)=0.61$ mag, $M_{\mathrm{ej}}=5 M_{\odot}$ and $E_{\mathrm{K}}=2 \times 10^{51} \mathrm{erg}$. Modjaz et al. (2008b) also derived a similar value, $R_{*}=1.1 \pm 0.46 R_{\odot}$ 


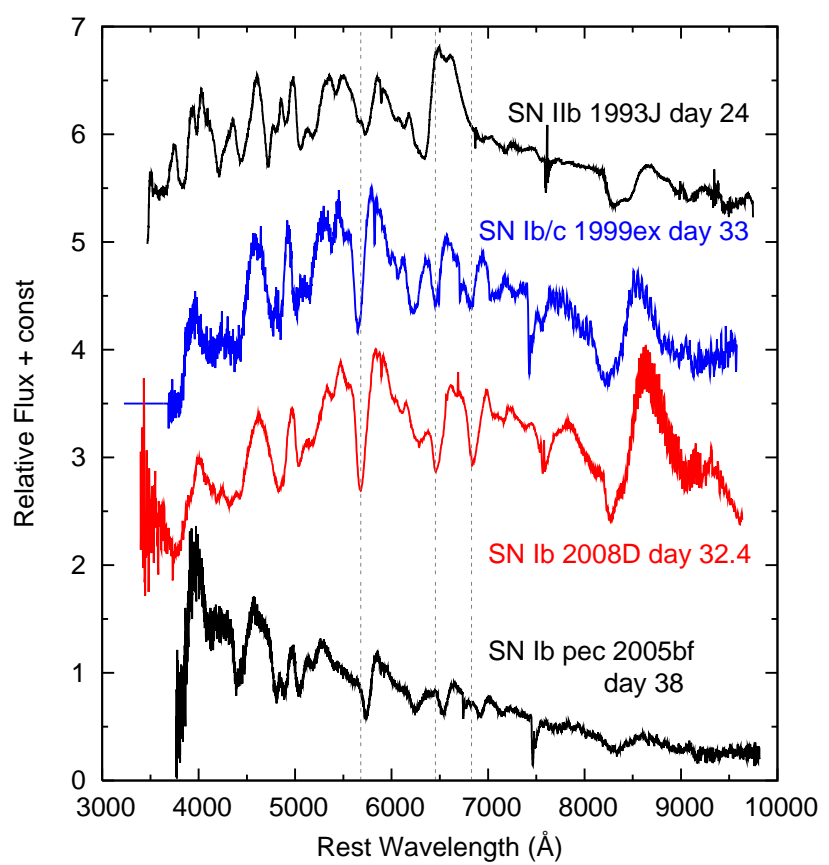

FIG. 9.- Spectral comparison among SNe 1993J (IIb; Barbon et al. 1995), 1999ex (Ib/c; Hamuy et al. 2002), 2008D (Ib) and 2005bf (peculiar Ib; Anupama et al. 2005). The epoch for SNe 1993J, 1999ex, and 2005bf is given in the estimated days from the explosion. Dashed, vertical lines show the position of the $\mathrm{He}$ lines (He I 5876, 6678, 7065) blueshifted with $v=10,000 \mathrm{~km} \mathrm{~s}^{-1}$. The spectra except for SN 2008D are taken from the SUSPECT database.

with $E(B-V)=0.6 \mathrm{mag}$ and the same $M_{\mathrm{ej}}$ and $E_{\mathrm{K}}$ with Soderberg et al. (2008). If $M_{\mathrm{ej}}$ and $E_{\mathrm{K}}$ derived in this paper are adopted, the estimated radius is $\sim 80 \%$ of their estimate. This is marginally consistent with the radius of model HE8 while it is smaller than HE6. In this sense, model HE8 seems to be more self-consistent. It must be noted, however, that Chevalier \& Fransson (2008) derived a larger radius, $R_{*} \sim 9 R_{\odot}$ by using the model by Chevalier (1992, and using the blackbody temperature and the radius presented by Soderberg et al. 2008).

Mazzali et al. (2008) estimated the ejecta parameters by modelling the bolometric LC and optical spectra. Their largest assumption is that a central remnant as massive as $\sim 3 M_{\odot}$ is implicitly assumed, which leads a massive $\mathrm{He}$ core mass $\left(\sim 10 M_{\odot}\right)$, and thus, a massive progenitor mass $\left(\sim 30 M_{\odot}\right)$. Our hydrodynamic/nucleosynthetic calculations show that a smaller central remnant is preferred $\left(\sim 1.6-1.8 M_{\odot}\right.$, Table 1$)$.

\subsection{SN 2008D in the Context of Type Ib/c Supernovae}

Figure 8 shows the kinetic energy of the ejecta and the ejected ${ }^{56} \mathrm{Ni}$ mass as a function of the estimated mainsequence mass for several core-collapse $\mathrm{SNe}$ (see, e.g., Nomoto et al. 2007). The parameters shown in Figure 8 are also listed in Table 2. SN 2008D is shown by a red circle in Figure 8. The ejecta parameters for other SNe shown in Figure 8 and Table 2 are derived from one-dimensional modelling as in this paper. Although there is a systematic uncertainty in the progenitor mass (Fig. 1), the progenitor mass of SNe shown in Figure 8 is estimated based on the $M_{\mathrm{MS}}-M_{\alpha}$ relation by Sugimoto
\& Nomoto (1980; used in Nomoto \& Hashimoto 1988) as in this paper. Thus, the relative position of $\mathrm{SNe}$ in the plots are robust.

The main-sequence mass of the progenitor of SN 2008D is estimated to be between normal SNe and GRB-SNe (or hypernovae). The kinetic energy of SN 2008D is also intermediate. Thus, SN 2008D is located between the normal SNe and the "hypernovae branch" in the $E_{\mathrm{K}}-$ $M_{\mathrm{MS}}$ diagram (upper panel of Fig. 8). The ejected ${ }^{56} \mathrm{Ni}$ mass in SN 2008D $\left(\sim 0.07 M_{\odot}\right)$ is similar to the ${ }^{56} \mathrm{Ni}$ masses ejected by normal $\mathrm{SNe}$ and much smaller than those in GRB-SNe.

Figure 9 compares the spectra of SNe 1993J (IIb, Barbon et al. 1995), 1999ex (Ib/c, Hamuy et al. 2002), 2005bf (Ib, Anupama et al. 2005, Tominaga et al. 2005, Folatelli et al. 2006), and 2008D (Ib). The epoch for SNe 1993J, 1999ex, and 2005bf is given in the estimated days from the explosion. The explosion epoch is uncertain up to $\sim 15$ days in SN 2005bf (Folatelli et al. 2006) while it is well constrained in SNe 1993J and 1999ex $(\lesssim 2$ days, Wheeler et al. 1993; Hamuy et al. 2002).

The spectra of SN 2008D and SN 1999ex are very similar (Valenti et al. 2008b), while SN 2005bf has a lower He velocities. Although the epoch of SN 2005bf is uncertain, the He line velocities in SN 2005bf is always lower than $8000 \mathrm{~km} \mathrm{~s}^{-1}$ (Tominaga et al. 2005). The He lines in SN 1993J are very weak at this epoch. The Fe features at $4500-5000 \AA$ are similar in these four $\mathrm{SNe}$, but those in SN 2005bf are narrower.

Malesani et al. (2009) suggested that the bolometric LCs of SNe 1999ex and 2008D are similar. If it is the case (although some discrepancy is shown by Modjaz et al. 2008b), the similarity in both the LC and the spectra suggests that SN 1999ex is located close to SN 2008D in the $E_{\mathrm{K}}-M_{\mathrm{MS}}$ and $M\left({ }^{56} \mathrm{Ni}\right)-M_{\mathrm{MS}}$ diagrams.

Comparison with other Type Ib SNe shown in Figure 8 is possible only for SN 2005bf although SN 2005bf is a very peculiar SN that shows a double peak LC with a very steep decline after the maximum, and increasing He line velocities (Anupama et al. 2005; Tominaga et al. 2005; Folatelli et al. 2006; Maeda et al. 2007). The LC of SN 2005bf is broader than that of SN 2008D, while the expansion velocity of SN 2005bf is lower than that of SN 2008D. These facts suggest that SN 2005bf is the explosion with lower $E_{\mathrm{K}} / M_{\mathrm{ej}}$ ratio (Table 2 ).

Malesani et al. (2009) also pointed the similarity of the LCs of SNe 1993J and 2008D. But the expansion velocity is higher in SN 2008D (see, e.g., Barbon et al. 1995; Prabhu et al. 1995). Thus, both the mass and the kinetic energy of the ejecta are expected to be smaller in SN 1993J. In fact, SN 1993J is explained by the explosion of a $4 M_{\odot}$ He core with a small mass H-rich envelope (Nomoto et al. 1993; Shigeyama et al. 1994; Woosley et al. 1994).

\section{CONCLUSIONS}

We presented a theoretical model for SN 2008D associated with the luminous X-ray transient 080109. Based on the progenitor models, hydrodynamics and explosive nucleosynthesis are calculated. Using the explosion models, radiative transfer calculations are performed. These models are tested against the bolometric LC and optical spectra. This is the first detailed model calculation for Type Ib SN that is discovered shortly after the explosion. 
We find that SN 2008D is a more energetic explosion than normal core-collapse SNe. We estimate that the ejecta mass is $M_{\mathrm{ej}}=5.3 \pm 1.0 M_{\odot}$ and the total kinetic energy of $E_{\mathrm{K}}=6.0 \pm 2.5 \times 10^{51} \mathrm{erg}$. The ejected ${ }^{56} \mathrm{Ni}$ mass is $\sim 0.07 M_{\odot}$. To eject the optimal amount of ${ }^{56} \mathrm{Ni}$, the mass of the central remnant is estimated to be $1.6-$ $1.8 M_{\odot}$. The error bars include only the uncertainty of the LC and spectral modelling.

Summing up the above masses, it is concluded that the progenitor star of SN $2008 \mathrm{D}$ has a $6-8 M_{\odot}$ He core prior to the explosion. There is essentially no $\mathrm{H}$ envelope with the upper limit of $5 \times 10^{-4} M_{\odot}$. Thus, the corresponding main-sequence mass of the progenitor is $M_{\mathrm{MS}}=20-25 M_{\odot}$ under the $M_{\mathrm{MS}}-M_{\alpha}$ relation by Sugimoto \& Nomoto (1980, used in Nomoto \& Hashimoto 1988). We note that there exist additional systematic uncertainties in this relation due to convection, mass loss, rotation, and binary effects. Our estimates of these masses and energy suggest that SN 2008D is near the bor- der between neutron star-forming and black hole-forming $\mathrm{SNe}$, and has properties intermediate between those of normal SNe and hypernovae associated with gamma-ray bursts.

M.T. and N.T. are supported by the JSPS (Japan Society for the Promotion of Science) Research Fellowship for Young Scientists. We have utilized the SUSPECT database. We would like to thank the contributors of the spectra used in the paper. This research has been supported in part by World Premier International Research Center Initiative (WPI Initiative), MEXT, Japan, and by the Grant-in-Aid for Scientific Research of the JSPS (10041110, 10304014, 11740120, 12640233, 14047206, $14253001,14540223,16740106,18104003,18540231$, 20540226) and MEXT (19047004, 20040004, 20041005, 07CE2002).

\section{REFERENCES}

Abbott, D. C., \& Lucy, L. B. 1985, ApJ, 288, 679

Anupama, G. C., et al. 2005, ApJ, 631, L125

Arnett, W.D. 1982, ApJ, 253, 785

Barbon, R., Benetti, S., Cappellaro, E., Patat, F., Turatto, M., \& Iijima, T. 1995, A\&AS, 110, 513

Berger, E., \& Soderberg, A. M. 2008, GCN Circ., 7159

Blinnikov, S., Lundqvist, P., Bartunov, O., Nomoto, K., \& Iwamoto, K. 2000, ApJ, 532, 1132

Branch, D., Jeffery, D. J., Young, T. R., \& Baron, E. 2006, PASP, 118,791

Chevalier, R. A. 1992, ApJ, 394, 599

Chevalier, R. A., \& Fransson, C. 2008, ApJ, 683, L135

Colella, P., \& Woodward, P.R. 1984, J. Comput. Phy. 54, 174

Deng, J., Tominaga, N., Mazzali, P. A., Maeda, K., \& Nomoto, K. 2005, ApJ, 624, 898

Deng, J., \& Zhu, Y. 2008, GCN Circ., 7160

Elmhamdi, A., Danziger, I. J., Branch, D., Leibundgut, B., Baron, E., \& Kirshner, R. P. 2006, A\&A, 450, 305

Folatelli, G, et al. 2006, ApJ, 641, 1039

Hachisu, I., Matsuda, T., Nomoto, K., \& Shigeyama, T. 1991, ApJ, 368, L27

Hamuy, M., et al. 2002, AJ, 124, 417

Hirschi, R., Meynet, G., \& Maeder, A. 2004, A\&A, 425, 649

Hix, W. R., \& Thielemann, F.-K. 1996, ApJ, 460, 869

Hix, W. R., \& Thielemann, F.-K. 1999, ApJ, 511, 862

Iwamoto, K., Nomoto, K., Hoflich, P., Yamaoka, H., Kumagai, S., \& Shigeyama, T. 1994, ApJ, 437, L115

Iwamoto, K., et al. 1998, Nature, 395, 672

Iwamoto, K., et al. 2000, ApJ, 534, 660

Kong, A. K.'H., \& Maccarone, T.J. 2008, ATel, 1355

Li, L.-X. 2008, MNRAS, 388, 603

Li, W., Filippenko, A. V. 2008, CBET, 1202, 1

Limongi, M., \& Chieffi, A. 2006, ApJ, 647, 483

Lucy, L. B. 1991, ApJ, 383, 308

Lucy, L. B. 1999, A\&A, 345, 211

Maeda, K., Nakamura, T., Nomoto, K., Mazzali, P. A., Patat, F., \& Hachisu, I. 2002, 565, 405

Maeda, K., Nomoto, K., Mazzali, P. A., \& Deng, J. 2006, ApJ, 640, 854

Maeda, K., et al. 2007, ApJ, 666, 1069

Maeda, K., et al. 2008, Science, 319, 1220

Magee, N. H., et al. 1995, in ASP Conf. Ser. 78., Astrophyical Applications of Powerful New Databases, ed. S. J. Adelman \& W. L. Wiese (San Francisco, CA :ASP), 51

Malesani, D., et al. 2009, 692, L84

Mazzali, P. A. \& Lucy, L.B. 1993, A\&A, 279, 447

Mazzali, P. A. 2000, A\&A, 363, 705

Mazzali, P. A., Iwamoto, K., \& Nomoto, K. 2000, ApJ, 545, 407

Mazzali, P. A., et al. 2002, ApJ, 572, L61

Mazzali, P. A., et al. 2003, ApJ, 599, L95

Mazzali, P. A., et al. 2005, Science, 308, 1284

Mazzali, P. A., et al. 2006a, ApJ, 645, 1323

Mazzali, P. A., et al. 2006b, Nature, 442, 1018

Mazzali, P. A., et al. 2008, Science, 321, 1185

Minezaki, T., et al. in preparation

Modjaz, M., Kirshner, R. P., Blondin, S., Challis, P., \& Matheson, T. 2008a, ApJ, 687, L9
Modjaz, M., et al. 2008b, submitted to ApJ (arXiv:0805.2201)

Modjaz, M., Chornock, R., Foley, R. J., Filippenko, A. V., \& Li, W. 2008c, CBET, 1221, 1

Nakamura, T., et al. 2001a, ApJ, 550, 991

Nakamura, T., Umeda, H., Iwamoto, K., Nomoto, K., Hashimoto, M., Hix, W. R., \& Thielemann, F.-K. 2001b, ApJ, 555, 880

Nomoto, K. 1982, ApJ, 253, 798

Nomoto, K., \& Hashimoto, M. 1988, Phys. Rep., 163, 13

Nomoto, K., Suzuki, T., Shigeyama, T., Kumagai, S., Yamaoka, H., \& Saio, H 1993, Nature, 364, 507

Nomoto, K., Hashimoto, M., Tsujimoto, T., Thielemann, F.-K., Kishimoto, N., Kubo, Y., \& Nakasato, N. 1997, Nucl. Phys. A, 616,79

Nomoto, K., Tominaga, N., Tanaka, M., Maeda, K., Suzuki, T., Deng, J. S., \& Mazzali, P.'A. 2007, SWIFT and GRBs: Unveiling the Relativistic Universe, Il Nuovo Cimento, 121, 1207-1222 (astroph/0702472)

Page, K. L., et al. 2008, GCN Report, 110.1

Prabhu, T. P., et al. 1995, A\&A, 295, 403

Quimby, R. M., Aldering, G., Wheeler, J. C., Höflich, P., Akerlof, C. W., \& Rykoff, E. S. 2007, ApJ, 668, L99

Rauscher, T., Heger, A., Hoffman, R. D., \& Woosley, S. E. 2002, ApJ, 576, 323

Shigeyama, T. \& Nomoto, K. 1990, ApJ, 360, 242

Shigeyama, T., Suzuki, T., Kumagai, S., Nomoto, K., Saio, H., \& Yamaoka, H. 1994, ApJ, 420, 341

Sauer, D. N., Mazzali, P. A., Deng, J., Valenti, S., Nomoto, K., \& Filippenko, A. V. 2006, MNRAS, 369, 1939

Soderberg, A. M. 2008, Nature, 453, 469

Sugimoto, D., \& Nomoto, K. 1980, Space Science Reviews, 25, 155

Tanaka, M., Maeda, K., Mazzali, P. A., \& Nomoto, K. 2007, ApJ, 668, L19

Tominaga, N., et al. 2005, ApJ, 633, L97

Tominaga, N. 2009, ApJ, 690, 526

Turatto, M., et al. 1998, ApJ, 498, L129

Valenti, S., Turatto, M., Navasardyan, H., Benetti, S., \& Cappellaro, E. 2008a, GCN Circ., 7163

Valenti, S., D'Elia, V., Della Valle, M., Benetti, S., Chincarini, G., Mazzali, P. A., \& Antonelli, L. A. 2008b, GCN Circ., 7221

Waxman, E., Mészáros, P., \& Campana, S. 2007, ApJ, 667, 351

Wellstein, S., \& Langer, N. 1999, A\&A, 350, 148

Wheeler, J. C., et al. 1993, ApJ, 417, L71

Woosley, S. E., Eastman, R. G., Weaver, T. A., \& Pinto, P. A 1994 ApJ, 429, 300

Xu, D., Zou, Y. C., \& Fan, Y. Z. 2008 (arXiv:0801.4325)

Yoshii, Y. 2002, in New Trends in Theoretical and Observational Cosmology, ed. K. Sato and T. Shiromizu (Tokyo: Universal Academy Press), 235

Yoshii, Y., Kobayashi, Y., \& Minezaki, T. 2003, BAAS, 202, 38.03

Zampieri, L., Pastorello, A., Turatto, M., Cappellaro, E., Benetti, S., Altavilla, G., Mazzali, P., \& Hamuy, M. 2003, MNRAS, 338, 711 

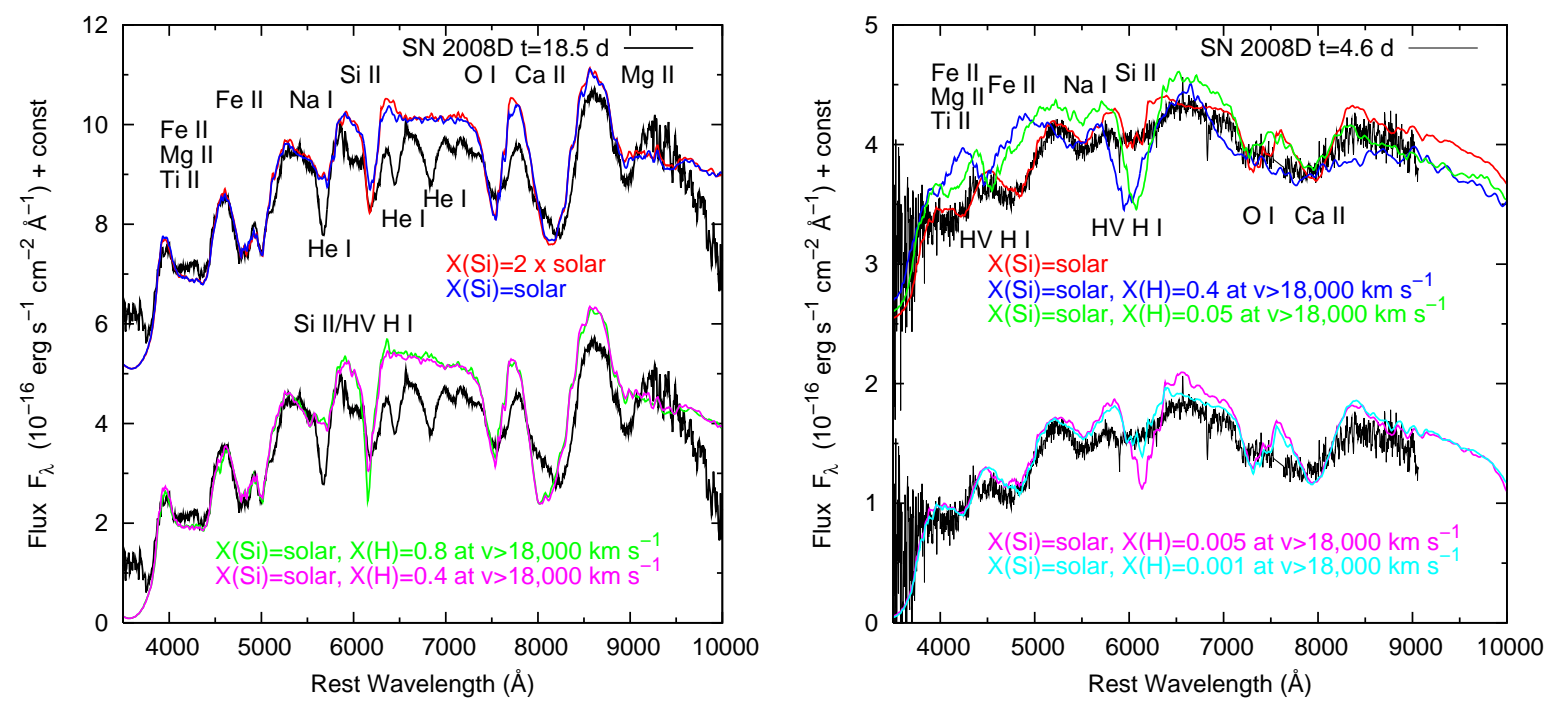

FIG. B1. - Left: Optical spectrum at $t=18.5$ days (Mazzali et al. 2008) compared with the synthetic spectra. The red and blue lines show the synthetic spectra with the models where the Si mass fraction is twice as large as the solar abundance (best fit) and the same as the solar abundance, respectively. The element abundances are assumed to be homogeneous in these models. The green and magenta lines show the synthetic spectra with $X(\mathrm{H})=0.8$ and 0.4 at $v>18000 \mathrm{~km} \mathrm{~s}^{-1}$, respectively. The corresponding mass of $\mathrm{H}$ is 0.4 and $0.2 M_{\odot}$, respectively. The homogeneous, solar abundance of $\mathrm{Si}$ is assumed in these models. Right: The optical spectrum at $t=4.6$ days (Mazzali et al. 2008) compared with the synthetic spectra. The red line shows the model with the solar abundance of Si, which gives a good fit to the observed spectrum. The green, blue, magenta and cyan lines show the models with $X(\mathrm{H})=0.4,0.05,0.005$ and 0.001 at $v>18000$ $\mathrm{km} \mathrm{s}^{-1}$, respectively. The corresponding mass of $\mathrm{H}$ is $0.4,0.025,0.0025$ and $0.0005 M_{\odot}$, respectively.

\section{APPENDIX}

\section{A. CONSTRUCTION OF BOLOMETRIC LiGHT CURVE}

The bolometric LC shown in this paper was constructed by using optical data taken by the MAGNUM telescope (Yoshii 2002; Yoshii, Kobayashi \& Minezaki 2003), the Himalayan Chandra Telescope, and Swift UVOT (U-band, Soderberg et al. 2008), and also NIR data taken by the MAGNUM telescope.

The bolometric luminosity was derived integrating the flux from $U$ (with the edge of $\left.9.68 \times 10^{14} \mathrm{~Hz}\right)$ to $K\left(1.00 \times 10^{14}\right.$ $\mathrm{Hz}$ ) band. The photometric points are interpolated by the third order natural spline. If the data point of a certain band is not available, we use linear interpolation of the magnitude.

The derived bolometric LC can be compared with that by Soderberg et al. (2008), Malesani et al. (2009), Modjaz et al. (2008b, $U-K_{S}$ integration) and Mazzali et al. (2008). Although the scatter up to 0.4 mag is found among the LCs by direct comparison, it is caused mainly by the difference in the assumed distance and reddening.

If the same distance and reddening are used (here we approximately correct the difference in the bolometric magnitude $\Delta M_{\mathrm{bol}}$ caused by the difference in the assumed reddening by $\Delta M_{\mathrm{bol}}=R_{V} \Delta E(B-V)$, where $R_{V}=3.1$ and $\Delta E(B-V)$ is the difference in the assumed color excess), the LCs around/after the maximum is consistent among those by this paper, Soderberg et al. (2008), Modjaz et al. (2008b) and Mazzali et al. (2008) within 0.1 mag, while the LC by Malesani et al. (2009) is fainter by 0.2-0.5 mag. For the pre-maximum epochs, the LCs by these papers are consistent within 0.2 mag except that the magnitude at $t=4$ days by this paper (shown by the arrow in the left panel of Fig. 3 ) is brighter than other ones by 0.25 mag.

Since the scatter in the maximum luminosity among the papers is up to $0.2 \mathrm{mag}$, it causes the uncertainty of the ejected ${ }^{56} \mathrm{Ni}$ mass up to $\sim 20 \%$. However, this uncertainty does not affect our determination of the ejecta mass because the change in the ${ }^{56} \mathrm{Ni}$ mass (and mass cut) is negligible compared to the ejecta mass ( $\$ 5$ ). In addition, the time scale of the bolometric LC around the maximum is reasonably consistent among the papers, the kinetic energy of the ejecta is also not affected.

\section{B. NONEXISTENCE OF THE HYDROGEN LAYERS}

Soderberg et al. (2008) identified the high velocity (HV) H $\alpha$ line for the absorption feature around $6150 \AA$ in the spectra around maximum. It is blended with the strong Si II line, and discrimination is not easy (e.g., Branch et al. 2006; Elmhamdi et al. 2006). The presence of $\mathrm{H}$ is important to specify the properties of the progenitor star just prior to the explosion. In addition, if the $\mathrm{H}$ layer is present, the estimate of $M_{\mathrm{ej}}$ and $E_{\mathrm{K}}$ may be affected since we have used bare He stars for the LC and spectral modelling.

First, we test the presence of $\mathrm{H}$ in the spectrum around maximum using model HE8. The left panel of Figure B1 shows the comparison between the observed spectrum at $t=18.5$ days (Mazzali et al. 2008) and synthetic spectra. The photospheric velocity at this epoch is $9000 \mathrm{~km} \mathrm{~s}^{-1}$ (Fig. 7). If the absorption at $6150 \AA$ is Si II $\lambda 6355$, the Doppler velocity of the absorption at $6150 \AA$ is $9300 \mathrm{~km} \mathrm{~s}^{-1}$, which is well consistent with the photospheric velocity. 
The red line shows the best fit model that includes Si twice as large as the solar abundance. The absorption is slightly shallower in the model with solar abundance Si (blue). Since the abundance twice as large as the solar abundance is reasonable for the middle layers of the ejecta, the $\mathrm{HV} \mathrm{H} \mathrm{I}$ is not necessarily required.

However, this does not exclude the possibility of the presence of $\mathrm{H}$ at the outer layers. If the absorption at $6150 \AA$ is $\mathrm{H} \alpha$, the Doppler velocity is $18500 \mathrm{~km} \mathrm{~s}^{-1}$. To test the presence of the $\mathrm{H}$ at such high velocity layers, we calculate model spectra by replacing He at $v>18000 \mathrm{~km} \mathrm{~s}^{-1}$ with $\mathrm{H}$. The green and magenta lines show the models with $X(\mathrm{H})=0.8$ and 0.4 at $v>18000 \mathrm{~km} \mathrm{~s}^{-1}$, respectively. The corresponding mass of $\mathrm{H}$ is 0.4 and $0.2 M_{\odot}$, respectively. The models also include the solar abundance of Si at at $v>9000 \mathrm{~km} \mathrm{~s}^{-1}$. While the model with $X(\mathrm{H})=0.8$ gives a too strong absorption, the model with $X(\mathrm{H})=0.4$ agree with the observed spectrum. Thus, the presence of $0.2 M_{\odot}$ of $\mathrm{H}$ cannot be denied from the spectrum around the maximum.

Next, we perform the similar tests using the very early spectrum. The right panel of Figure B1 shows the comparison between the observed spectrum at $t=4.6$ days (Mazzali et al. 2008) and the synthetic spectra. The red line shows the best fit model, which include the solar abundance of Si. The blue line shows the model that have the solar abundance of $\mathrm{Si}$ and $X(\mathrm{H})=0.4$ at $v>18000 \mathrm{~km} \mathrm{~s}^{-1}$. Although this model gives a reasonable fit to the spectrum at $t=18.5$ days (left panel of Fig. B1), it shows too strong $\mathrm{H} \alpha$ and $\mathrm{H} \beta$ at $t=4.6$ days (the lack of $\mathrm{H} \beta$ has been pointed out by Malessani et al. 2009). We get the stronger line at earlier epochs because the density at the high velocity layers $\left(v=18000 \mathrm{~km} \mathrm{~s}^{-1}\right)$ becomes lower with time, and the line forming there is more effective at earlier epochs.

The green, magenta and cyan lines show the models with smaller mass fraction of $\mathrm{H}, X(\mathrm{H})=0.05,0.005$, and 0.001 , respectively. The corresponding mass of $\mathrm{H}$ is $0.025,0.0025$ and $0.0005 M_{\odot}$. The models with $X(\mathrm{H})=0.05$ and 0.005 (green and magenta) still shows too strong $\mathrm{H}$ I lines. With $X(\mathrm{H})=0.001$, the $\mathrm{H} \alpha$ line has little effect on the absorption at $6150 \AA$ although the model spectrum still has a sharp absorption of the $\mathrm{HV} \mathrm{H} \alpha$.

If we use model HE6, the mass at the outer layers is smaller. Thus, we conclude that the mass of $\mathrm{H}$ is smaller than $5 \times 10^{-4} M_{\odot}$. 